\title{
FUNCTORIALITY AND THE INVERSE GALOIS PROBLEM II: GROUPS OF TYPE $B_{n}$ AND $G_{2}$
}

\author{
CHANDRASHEKHAR KHARE, MICHAEL LARSEN, AND GORDAN SAVIN
}

\section{INTRODUCTION}

1.1. Earlier work. Let $\ell$ be a prime. In our previous work [KLS], which generalised a result of Wiese [W], the Langlands functoriality principle was used to show that for every positive integer $t$ there exists a positive integer $k$ divisible by $t$ such that the finite simple group $\mathrm{C}_{n}\left(\ell^{k}\right)=\operatorname{PSp}_{2 n}\left(\mathbb{F}_{\ell^{k}}\right)$ is the Galois group of an extension of $\mathbb{Q}$ unramified outside $\{\infty, \ell, q\}$ where $q \neq 2$ is a prime that depends on $t$. The construction is based on the following three steps.

(1) Starting with a cuspidal automorphic representation on the split group $\mathrm{SO}_{2 n+1}$ constructed using the Poincaré series, we use the global lift of Cogdell, Kim, PiatetskiShapiro and Shahidi [CKPS] and results of Jiang and Soudry [JS1] to obtain a self-dual cuspidal automorphic representation $\Pi$ of $G_{2 n}(\mathbb{A})$, with $\mathbb{A}$ the adeles of $\mathbb{Q}$, such that the following three conditions hold:

- $\Pi_{\infty}$ is cohomological.

- $\Pi_{q}$ is a supercuspidal representation of depth 0 .

- $\Pi_{v}$ is unramified for all primes $v \neq \ell, q$.

(2) The work of Kottwitz, Clozel, Harris-Taylor and Taylor-Yoshida yields the following theorem (see Theorem 3.6 of $[\mathrm{Ty}]$ or Theorem 1.1 of [Ha] $)$. We use the conventions and notations of $\S 1$ of [Ha].

Theorem 1.1. Let $\Pi$ be a self-dual cuspidal automorphic representation $\Pi$ of $\mathrm{GL}_{m}(\mathbb{A})$ such that $\Pi_{\infty}$ is cohomological. Assume that for some finite place $v_{0}$ of $\mathbb{Q}, \Pi_{v_{0}}$ is square integrable. Then attached to $\pi$ and a choice of an embedding $\iota: \overline{\mathbb{Q}} \hookrightarrow \overline{\mathbb{Q}}_{\ell}$, there is an irreducible $\ell$-adic representation $r_{\Pi}^{\prime}: G_{\mathbb{Q}} \rightarrow \mathrm{GL}_{m}\left(\overline{\mathbb{Q}}_{\ell}\right)$ of the Galois group $G_{\mathbb{Q}}$ of $\mathbb{Q}$ such that for all primes $v$ of $\mathbb{Q}$ of residue characteristic $\neq \ell$ we have:

$$
W D_{v}\left(r_{\Pi}^{\prime}\right)^{\text {Frob-ss }}=\mathcal{L}\left(\Pi_{v} \otimes||^{\frac{1-m}{2}}\right)
$$

Here $W D_{v}\left(r_{\Pi}^{\prime}\right)$ is the Weil-Deligne parameter of $\left.r_{\Pi}^{\prime}\right|_{D_{v}}$ with $D_{v}$ a decomposition group at $v, \mathcal{L}$ is the normalised local Langlands correspondence, and Frob-ss denotes Frobenius semisimplification.

CK was partially supported by NSF grants DMS 0355528 and DMS 0653821, the Miller Institute for Basic Research in Science, University of California Berkeley, and a Guggenheim fellowship.

ML was partially supported by NSF grant DMS 0354772.

GS was partially supported by NSF grant DMS 0551846. 
Remark: Let $\chi_{\ell}$ be the $\ell$-adic cyclotomic character. If $m=2 n+1$ we consider a twist $r_{\Pi}=r_{\Pi}^{\prime} \otimes \chi_{\ell}^{n}$ and note that we have

$$
W D_{v}\left(r_{\Pi}\right)^{\text {Frob-ss }}=\mathcal{L}\left(\Pi_{v}\right) .
$$

(3) The last step consists of reducing $r_{\Pi}^{\prime}$ modulo $\ell$. The parameter of $\Pi_{q}$ can be picked so that $r_{\Pi}\left(G_{\mathbb{Q}_{q}}\right)$ is a metacyclic group deeply embedded in $r_{\Pi}^{\prime}\left(G_{\mathbb{Q}}\right)[\mathrm{KW}$. That is, for some large positive integer $d, r_{\Pi}^{\prime}\left(G_{\mathbb{Q}_{q}}\right)$ is contained in every normal subgroup of $r_{\Pi}^{\prime}\left(G_{\mathbb{Q}}\right)$ of index less than or equal to $d$. This property is crucial to assure, using the main result of $\left[\mathrm{LP}\right.$, that the reduction modulo $\ell$ is a simple group of type $\operatorname{PSp}_{2 n}\left(\mathbb{F}_{\ell^{k}}\right)$.

1.2. Main theorem. The purpose of this work is to extend these results and to construct finite simple groups of type $\mathrm{B}_{n}$ and $\mathrm{G}_{2}$ as Galois groups over $\mathbb{Q}$. In the case of $\mathrm{G}_{2}$, our result depends on a recent technical improvement of Theorem 1.1 due to Shin [Sh] in the case that $m$ is odd. He shows that we may drop the hypothesis of the existence of a place $v_{0}$ such that $\Pi_{v_{0}}$ is square integrable. The resulting representation $r_{\Pi}^{\prime}$ is semi-simple although it is expected to be irreducible.

We can state our main theorem:

Theorem 1.2. Let $t$ be a positive integer. We assume that $t$ is even if $\ell=3$ in the first case below:

(1) Let $\ell$ be a prime. Then there exists an integer $k$ divisible by $t$ such that the simple group $\mathrm{G}_{2}\left(\mathbb{F}_{\ell^{k}}\right)$ appears as a Galois group over $\mathbb{Q}$.

(2) Let $\ell$ be an odd prime. Then there exists an integer $k$ divisible by $t$ such that the finite simple group $\mathrm{SO}_{2 n+1}\left(\mathbb{F}_{\ell^{k}}\right)^{\text {der }}$ or the finite classical group $\mathrm{SO}_{2 n+1}\left(\mathbb{F}_{\ell^{k}}\right)$ appears as a Galois group over $\mathbb{Q}$.

(3) If $\ell \equiv 3,5(\bmod 8)$, then there exists an integer $k$ divisible by $t$ such that the finite simple group $\mathrm{SO}_{2 n+1}\left(\mathbb{F}_{\ell^{k}}\right)^{\text {der }}$ appears as a Galois group over $\mathbb{Q}$.

1.3. Sketch of proof. The construction of Galois groups in Theorem 1.2 is based on the functorial lift from $\mathrm{Sp}_{2 n}$ to $\mathrm{GL}_{2 n+1}$ CKPS plus the lift from $\mathrm{G}_{2}$ to $\mathrm{Sp}_{6}$ using the theta correspondence arising from the minimal representation of the exceptional group $\mathrm{E}_{7}$ (see Sa1 for a definition of the minimal representation). The main new technical difficulty in implementing the strategy of [KLS in the present case, is that $\mathrm{GL}_{2 n+1}\left(\mathbb{Q}_{p}\right)$ has self-dual supercuspidal representations only if $p=2$. Thus, while we can still construct a self-dual cuspidal automorphic representation $\Pi$ of $\mathrm{GL}_{2 n+1}$ which should give rise to our desired Galois groups, the local component $\Pi_{q}$ cannot be supercuspidal. For groups of type $\mathrm{B}_{n}$ we can remedy the situation by requiring that the local component $\Pi_{2}$ be supercuspidal (which we pick to be of depth one). Existence of a global $\Pi$ with such local component $\Pi_{2}$ is again obtained using the global lift from $\mathrm{Sp}_{2 n}$ plus recently announced backward lift from $\mathrm{GL}_{2 n+1}$ to $\mathrm{Sp}_{2 n}$ by Jiang and Soudry [JS2]. The local component $\Pi_{2}$ not only assures us of the existence of the $\ell$-adic representation $r_{\Pi}$, without using new results of Shin, but it also gives us a certain control over the Galois group obtained by reducing $r_{\Pi}$ modulo $\ell$. More precisely, $\Pi_{2}$ can be picked so that the image of the local Langlands parameter is a finite group $I$ in $\mathrm{GL}_{2 n+1}(\mathbb{C})$ with the following properties:

- $I /[I, I] \cong \mathbb{Z} /(2 n+1) \mathbb{Z}$.

- $[I, I] \cong(\mathbb{Z} / 2 \mathbb{Z})^{2 n}$. 
If $\ell \equiv 3,5(\bmod 8)$ then the first property of $I$ implies that the Galois group is $\mathrm{SO}_{2 n+1}\left(\mathbb{F}_{\ell^{k}}\right)^{\text {der }}$ and not $\mathrm{SO}_{2 n+1}\left(\mathbb{F}_{\ell^{k}}\right)$. If $n=3$ then the second property of $I$ implies that $\Pi_{2}$ is not a lift from $\mathrm{G}_{2}\left(\mathbb{Q}_{2}\right)$ and the Galois group is not $\mathrm{G}_{2}\left(\ell^{k}\right)$.

Correction to [KLS] : With the definition of the group of type $(n, p)$ in [KLS], to ensure that $\bar{\rho}\left(D_{q}\right)$ in $\S 5.2$ be of type $(n, p)$ we should ask that the $K$ of $\S 3.3$ also contain $\mathbb{Q}\left(\zeta_{\ell}\right)$, in addition to the other conditions there. Alternatively, and better, the definition of a group of type $(n, p)$ in [KLS] could be modified (and made less restrictive) as in Definition 10.2 below. All statements in [KLS] then go through with this altered definition, with obvious modifications in their proof.

Acknowledgments: We would like to thank Dick Gross and Guy Henniart for helping us with irreducible supercuspidal parameters and Mark Reeder for his help with small representations of reductive groups.

\section{LOCAL DISCRETE SERIES PARAMETERS}

Let $k$ be a local field and $G$ a connected reductive and split group over $k$. Conjecturally, representations of $G(k)$ correspond to (certain) homomorphisms of the Weil-Deligne group

$$
\phi: W D_{k} \rightarrow G^{*}(\mathbb{C})
$$

into the Langlands dual group $G^{*}(\mathbb{C})$. In this paper we shall be concerned with the following cases:

\begin{tabular}{|c||c|c|c|c|}
\hline$G$ & $\mathrm{GL}_{n}$ & $\mathrm{Sp}_{2 n}$ & $\mathrm{PGSp}_{6}$ & $\mathrm{G}_{2}$ \\
\hline$G^{*}$ & $\mathrm{GL}_{n}$ & $\mathrm{SO}_{2 n+1}$ & $\operatorname{Spin}_{7}$ & $\mathrm{G}_{2}$ \\
\hline
\end{tabular}

If $G=\mathrm{Sp}_{2 n}$, it will be convenient to realize the dual group as $\mathrm{SO}(U)$ for some choice of a non-degenerate complex orthogonal space $U$ of dimension $2 n+1$. Then a discrete series parameter for $\operatorname{Sp}_{2 n}(k)$ is a homomorphism $\phi: W D_{k} \rightarrow \mathrm{SO}(U)$ such that under the action of $W D_{k}$ the orthogonal space $U$ decomposes into irreducible summands

$$
U=U_{1} \oplus \cdots \oplus U_{s}
$$

where each $U_{i}$ is a non-degenerate orthogonal subspace of $U$. Moreover, if $\phi_{i}$ denotes the representation of $W D_{k}$ on $U_{i}$, then $\phi_{i} \cong \phi_{j}$ if and only if $i=j$. In other words, we are requiring that the image of $W D_{k}$ is not contained in a proper Levi factor in $G^{*}$.

Consider now the case $k=\mathbb{R}$. In this case the Weil-Deligne group is the same as the Weil group $W_{\mathbb{R}}$. For every non-zero integer $a$ let $\eta_{a}$ be a character of $W_{\mathbb{C}} \cong \mathbb{C}^{\times}$defined by

$$
\eta_{a}(z)=\left(\frac{z}{\bar{z}}\right)^{a}
$$

Let

$$
\phi(a)=\operatorname{Ind}_{W_{\mathbb{C}}}^{W_{\mathbb{R}}} \eta_{a} .
$$

This is an irreducible and orthogonal 2 dimensional representation of $W_{\mathbb{R}}$. Its determinant is the unique non-trivial quadratic character $\chi_{\infty}$ of $W_{\mathbb{R}}^{a b} \cong \mathbb{R}^{\times}$. If $a_{1}, \ldots, a_{n}$ are non-zero integers such that $a_{i} \neq \pm a_{j}$, then

$$
\phi\left(a_{1}, \ldots, a_{n}\right) \oplus \chi_{\infty}^{n} .
$$


is a discrete series parameter for the group $\operatorname{Sp}_{2 n}(\mathbb{R})$. Note that the choice of exponent - $n$ in the last summand is made so that the image of the parameter is contained in $\mathrm{SO}_{2 n+1}(\mathbb{C})$. Note that the parameter is determined by $a_{i}$ up to permutation of indices and change of signs. If $n=3$, then the image of the parameter is contained in $\mathrm{G}_{2}(\mathbb{C}) \subset \mathrm{SO}_{7}(\mathbb{C})$ if and only if

$$
a_{1}+a_{2}+a_{3}=0
$$

for some choices of signs of $a_{i}$ 's. Let $\sigma_{\infty}$ be a generic discrete series representation of $\operatorname{Sp}_{2 n}(\mathbb{R})$ (or of $\mathrm{G}_{2}(\mathbb{R})$ ) corresponding to this parameter.

Let $\Pi_{\infty}$ be the lift of $\sigma_{\infty}$ to $\mathrm{GL}_{2 n+1}(\mathbb{R})$. The infinitesimal character of $\Pi_{\infty}$ is represented by a $2 n+1$-tuple

$$
\left(a_{1}, \ldots, a_{n},-a_{1}, \ldots,-a_{n}, 0\right) .
$$

In particular, $\Pi_{\infty}$ is cohomological, as defined by Clozel [Cl].

\section{DePth ZERo GENERIC SUPERCUSiPdAL REPRESENTATIONS}

Let $q$ be an odd prime. Let $\Omega_{q^{\prime}}$ denote the set of all complex roots of unity of order prime to $q$. The Frobenius acts on $\Omega_{q^{\prime}}$ by

$$
F(\tau)=\tau^{q}
$$

for every $\tau$ in $\Omega_{q^{\prime}}$. Note that all $F$-orbits are finite. These orbits play a key role in the description of tame parameters.

Lemma 3.1. Let $\tau$ be a root of 1 different from \pm 1 . Assume that the F-orbit of $\tau$ has $m$ different elements:

$$
\tau, \tau^{q}, \ldots, \tau^{q^{m-1}} .
$$

If $\tau^{-1}$ is on this list, that is, if $\tau^{-1}=\tau^{q^{n}}$ for some $n<m$ then $m=2 n$.

Proof. First of all, note that $0<n$ since $\tau \neq \pm 1$. Raising $\tau^{-1}=\tau^{q^{n}}$ to the $q^{n}$-th power gives $\tau=\tau^{q^{2 n}}$. Since $\tau=\tau^{q^{k}}$ if and only if $k$ is a multiple of $m$, and $0<2 n<2 m$, it follows that $m=2 n$, as claimed.

We are now ready to define irreducible tame self-dual parameters of $\operatorname{Sp}_{2 n}\left(\mathbb{Q}_{q}\right)$. Let $\mathbb{Q}_{q^{2 n}}$ be the unique unramified extension of $\mathbb{Q}_{q}$ of degree $2 n$. Then

$$
\mathbb{Q}_{q^{2 n}}^{\times}=\langle q\rangle \times \mathbb{F}_{q^{2 n}}^{\times} \times U_{1}
$$

where $U_{1}$ is the maximal pro $q$-subgroup of $\mathbb{Q}_{q^{2 n}}^{\times}$. A character of $\mathbb{Q}_{q^{2 n}}^{\times}$is called tame if it is trivial on $U_{1}$. Let $\zeta_{2 n}$ be a primitive root in $\mathbb{F}_{q^{2 n}}^{\times}$. Pick $\tau$, a complex root of 1 such that the $F$-orbit $\tau, \tau^{q}, \ldots$ has precisely $2 n$ distinct elements and $\tau^{q^{n}}=\tau^{-1}$. (For example, $\tau$ can be picked a primitive root of order $q^{n}+1$.) Then $\tau$ defines a tame character $\eta$ of $\mathbb{Q}_{q^{2 n}}^{\times}$by

$$
\left\{\begin{array}{l}
\eta\left(\zeta_{2 n}\right)=\tau \\
\eta(q)=1
\end{array}\right.
$$

Let $W_{\mathbb{Q}_{q}}$ and $W_{\mathbb{Q}_{q} 2 n}$ be the local Weil groups of $\mathbb{Q}_{q}$ and $\mathbb{Q}_{q^{2 n}}$. Recall that

$$
W_{\mathbb{Q}_{q}} / W_{\mathbb{Q}_{q} 2 n} \cong \operatorname{Gal}\left(\mathbb{F}_{q^{2 n}} / \mathbb{F}_{q}\right) \text {. }
$$


Via the local class field theory we have an identification $W_{\mathbb{Q}_{q^{2 n}}^{a b}} \cong \mathbb{Q}_{q^{2 n}}^{\times}$. Note that $\eta \circ F^{i} \neq \eta$ for $1<i \leq 2 n$ and $\eta \circ F^{n}=\bar{\eta}$. In particular, the character $\eta$ defines an irreducible, orthogonal $2 n$-dimensional representation

$$
\phi(\tau)=\operatorname{Ind}_{W_{\mathbb{Q}_{q} 2 n}}^{W_{\mathbb{Q}_{q}}}(\eta)
$$

of $W_{\mathbb{Q}_{q}}$. We note that the determinant of $\phi(\tau)$ is the unique unramified quadratic character $\chi_{q}$ of $W_{\mathbb{Q}_{q}}^{a b} \cong \mathbb{Q}_{q}^{\times}$.

Pick a sequence $\tau_{1}, \ldots, \tau_{s}$ of roots in $\Omega_{q^{\prime}}$ belonging to different $F$-orbits of order $2 n_{1}, \ldots, 2 n_{s}$ such that $\tau^{q^{n_{i}+1}}=1$ for every $i$ and $2 n_{1}+\cdots+2 n_{s}=2 n$. Corresponding to this we have a tame regular discrete series parameter for the split group $\operatorname{Sp}_{2 n}\left(\mathbb{Q}_{q}\right)$

$$
\phi=\phi\left(\tau_{1}, \ldots, \tau_{s}\right) \oplus \chi_{q}^{s}
$$

where, as in the case of real groups, the exponent $d$ is picked to assure that the image of the parameter is contained in $\mathrm{SO}_{2 n+1}(\mathbb{C})$. Note that the image $\phi\left(I_{q}\right)$ of the inertia subgroup $I_{q} \subseteq W_{\mathbb{Q}_{q}}$ is contained in a maximal torus of $\mathrm{SO}_{2 n+1}(\mathbb{C})$ and $\phi(F)$ is an elliptic element of the Weyl group. If $s=1$, for example, then the image of the inertia is a cyclic group generated by an element whose eigenvalues are

$$
\tau, \tau^{q}, \ldots, \tau^{q^{n}}, \tau^{-1}, \ldots, \tau^{-q^{n}}, 1
$$

and $\phi(F)$ correspond to the Coxeter element in the Weyl group.

Proposition 3.2. The image of a tame regular discrete series parameter $\phi=\phi\left(\tau_{1}, \ldots, \tau_{s}\right) \oplus$ $\chi_{q}^{s}$ of $\mathrm{Sp}_{6}\left(\mathbb{Q}_{q}\right)$ is contained in $\mathrm{G}_{2}(\mathbb{C})$ if and only if one of the two holds:

(1) $s=3$ and $\tau_{1} \tau_{2} \tau_{3}=1$, for some choices of $\tau_{i}^{ \pm}$(F-orbit of $\tau_{i}$ consists of $\tau_{i}$ and $\left.\tau_{i}^{-1}\right)$.

(2) $s=1$ and $\tau$ satisfies $\tau^{q^{2}-q+1}=1$. (Recall that $\tau$, a priori, satisfies a weaker condition $\tau^{q^{3}+1}=1$.)

Proof. The weights of the 7-dimensional representation of $\mathrm{G}_{2}(\mathbb{C})$ are 0 and six short roots. Pick three short roots $\alpha_{1}, \alpha_{2}$ and $\alpha_{3}$ such that $\alpha_{1}+\alpha_{2}+\alpha_{3}=0$. If $t$ is a semi-simple element in $\mathrm{G}_{2}(\mathbb{C})$, put $\lambda_{i}^{ \pm}= \pm \alpha_{i}(t)$. Then $\lambda_{1}^{ \pm}, \lambda_{2}^{ \pm}, \lambda_{3}^{ \pm}$and 1 are the eigenvalues of $t$ in the 7-dimensional representation. Note that $\lambda_{1} \lambda_{2} \lambda_{3}=1$.

If the parameter $\phi$ is contained in $\mathrm{G}_{2}(\mathbb{C})$ then $\phi(F)$ corresponds to a Weyl group element in $\mathrm{G}_{2}$ of even order. Since 2 and 6 are only even orders of elements in the Weyl group of $\mathrm{G}_{2}$, we see that $s=1$ or 3 . If $s=3$ then $\phi(F)$ corresponds to -1 in the Weyl group and the condition $\lambda_{1} \lambda_{2} \lambda_{3}=1$ translates into $\tau_{1} \tau_{2} \tau_{3}=1$. If $s=1$ then $\phi(F)$ corresponds to the Coxeter element. We can pick the Coxeter element (or alternatively the roots $\alpha_{i}$ ) so that it cyclically permutes the roots

$$
\alpha_{1},-\alpha_{2}, \alpha_{3},-\alpha_{1}, \alpha_{2},-\alpha_{3} .
$$

On the other hand, $\phi\left(I_{q}\right)$ is generated by a semi-simple element $t$ with non-trvial eigenvalues $\tau, \tau^{q}, \tau^{q^{2}}, \tau^{-1}, \tau^{-q}, \tau^{-q^{2}}$ which $\phi(F)$ permutes cyclically in the given order. In particular, the condition $\lambda_{1} \lambda_{2} \lambda_{3}=1$ translates into $\tau^{1-q+q^{2}}=1$, as desired. Conversely, if the parameter satisfies the conditions of $(1)$ and $(2)$ then we can factor $\phi$ through $\mathrm{G}_{2}(\mathbb{C})$ since -1 and the Coxeter element can be lifted from the Weyl group to $\mathrm{G}_{2}(\mathbb{C})$. 
Let $\sigma_{q}$ be $a$ generic supercuspidal representation of $\mathrm{Sp}_{2 n}\left(\mathbb{Q}_{q}\right)$ (or of $\mathrm{G}_{2}\left(\mathbb{Q}_{q}\right)$ ) corresponding, via DeBacker-Reeder, to a tame parameter as above. Then the lift of $\sigma_{q}$ to $\mathrm{GL}_{2 n+1}\left(\mathbb{Q}_{q}\right)[\mathrm{Sa2}$ ] is

$$
\Pi_{1} \times \cdots \times \Pi_{s} \times \chi_{q}^{s}
$$

This is a tempered representation parabolically induced from supercuspidal representations $\Pi_{1}, \ldots, \Pi_{s}$ corresponding to irreducible tame paramters $\phi\left(\tau_{1}\right), \ldots, \phi\left(\tau_{s}\right)$ by the local Langlands correspondence [HT]. We note that the recipe of DeBacker-Reeder [DR] involves picking a hyperspecial compact subgroup of $\operatorname{Sp}_{2 n}\left(\mathbb{Q}_{p}\right)$. Since there are two non-conjugate hyperspecial maximal compact subgroups here, there are two possible $\sigma_{q}$. They have the same lift to $\mathrm{GL}_{2 n+1}\left(\mathbb{Q}_{p}\right)$.

Of interest to us is the parameter of type $\phi(\tau) \oplus \chi_{q}$ where $q$ and $\tau$ are picked using the following lemma (Lemma 3.4 in [KLS]):

Lemma 3.3. Given a positive integer $m=2 n$, a prime $\ell$, a finite Galois extension $K$ of $\mathbb{Q}$, and positive integers $t$ and $d$, there exists odd primes $p$ and $q$ such that

(1) Primes $\ell, p$ and $q$ are all distinct.

(2) The prime $p$ is greater than $d$.

(3) If $\mathrm{SO}_{2 n+1}\left(\mathbb{F}_{\ell^{k}}\right)$ contains an element of order $p$ then $\mathbb{F}_{\ell^{k}}$ contains $\mathbb{F}_{\ell^{t}}$. In particular, $t$ divides $k$.

(4) The prime $q$ splits completely in $K$.

(5) The order of $q$ in $\mathbb{F}_{p}^{\times}$is exactly $m$.

We remark that if $n=3, \ell=3$, and $t$ is even, then no Ree group ${ }^{2} \mathrm{G}_{2}\left(\mathbb{F}_{3^{2 f+1}}\right)$ contains an element of order $p$. Indeed,

$$
{ }^{2} \mathrm{G}_{2}\left(\mathbb{F}_{3^{2 f+1}}\right)<\mathrm{G}_{2}\left(\mathbb{F}_{3^{2 f+1}}\right)<\mathrm{SO}_{7}\left(\mathbb{F}_{3^{2 f+1}}\right),
$$

and $t$ does not divide $2 f+1$.

Let $K$ be the composite of all Galois extensions of $\mathbb{Q}$ of degree $\leq d$ and ramified at $2, \ell$ and no other primes. This is a finite degree Galois extension of $\mathbb{Q}$ ramified at $2, \ell$ and no other primes. Let $p$ and $q$ be the primes given by Lemma 3.3 applied to this field $K$. Let $\tau$ be a primitive $p$-th root of 1 . Since the order of $q$ in $\mathbb{F}_{p}^{\times}$is precisely $2 n$, the $\operatorname{Fr}_{q}$-orbit of $\tau$ gives rise to a tame parameter $\phi(\tau) \oplus \chi_{q}$. Moreover, if $n=3$ then $\tau^{q^{2}-q+1}=1$ since $\tau$ is of order $p$ and $p$, by construction, divides $\Phi_{6}(q)=q^{2}-q+1$. In particular, this parameter is automatically a $\mathrm{G}_{2}$-parameter. In any case, we note that the image of the inertia $I_{q}$ subgroup is a cyclic group of order $p$. The image of the Weil group is a semi-direct product of the cyclic group $\mathbb{Z} / p \mathbb{Z}$ and the cyclic group $\mathbb{Z} / 2 n \mathbb{Z}$. This group is also called a metacyclic group and denoted by $\Gamma_{2 n, p}$.

\section{IRREDUCIBLE SUPERCUSPIDAL PARAMETERS}

As we have seen in the previous section, the image of a tame supercuspidal parameter $\varphi: W_{k} \rightarrow G^{*}$ is not irreducible when acting on the standard representation $U$ of $G^{*}$. In particular, the lift to $\mathrm{GL}_{n}(k)(n=\operatorname{dim}(U))$ of the corresponding supercuspidal representation is not supercuspidal. In order to remedy this, we need to introduce certain wildly ramified parameters. This will be done using (so-called) Jordan subgroups of the complex reductive group $G^{*}$. A Jordan subgroup $J$ of $G^{*}$ is an elementary abelian $p$-subgroup such that its 
normalizer $N$ in $G^{*}$ is a finite subgroup and $J$ is a minimal normal subgroup of $N$ see $[\mathrm{KT}$, page 505. The following is a partial list of Jordan subgroups.

\begin{tabular}{|c|c|c|}
\hline$G^{*}$ & $J$ & $N / J$ \\
\hline $\mathrm{SO}_{2 n+1}$ & $\left(\mathbb{F}_{2}\right)^{2 n}$ & $S_{2 n+1}$ \\
$\mathrm{G}_{2}$ & $\left(\mathbb{F}_{2}\right)^{3}$ & $\mathrm{SL}_{3}(2)$ \\
\hline
\end{tabular}

Here $S_{2 n+1}$ is the symmetric group of $2 n+1$ letters. We note that the conjugation action of $N / J$ on $J$ given by the standard representation of $N / J$ on $J$. (In the first case we mean by this the restriction of the permutation representation of $S_{2 n+1}$ on $\mathbb{F}_{2}^{2 n+1}$ to the hyperplane given by $\sum_{i=1}^{2 n+1} x_{i}=0$.) However the extension of $N / J$ by $J$ is not necessarily split.

We shall now construct a map $\varphi: W_{\mathbb{Q}_{p}} \rightarrow G^{*}$ such that the image of the wild inertia is $J$ (in particular $p=2$ ) and the image of $W_{\mathbb{Q}_{p}}$ is an intermediate subgroup $J \subseteq I \subseteq N$ acting irreducibly on the standard representation of $G^{*}$.

Let us consider the case $G^{*}=\mathrm{SO}_{2 n+1}(\mathbb{C})$ first. Let us abbreviate $m=2 n+1$, and let $\mathbb{Q}_{2^{m}}$ be the unramified extension of $\mathbb{Q}_{2}$ of degree $m$. Then

$$
\mathbb{Q}_{2^{m}}^{\times}=\langle 2\rangle \times \mathbb{F}_{2^{m}}^{\times} \times U
$$

where $U$ is a pro-2 group with a filtration $U \supset U_{1} \supset U_{2} \ldots$ such that $U / U_{1} \cong \mathbb{F}_{2^{m}}$. Let $e$ be a primitive element in $\mathbb{F}_{2^{m}}$. Let $e_{i}=\mathrm{Fr}_{2}^{i-1}(e)$. Then $e=e_{1}, e_{2}, \ldots, e_{m}$ give a basis of $\mathbb{F}_{2^{m}}$ over $\mathbb{F}_{2}$. In particular, we have fixed an isomorphism

$$
U / U_{1} \cong\left(\mathbb{F}_{2}\right)^{m} \text {. }
$$

In this way any character of $U / U_{1}$ can be viewed as an $m$-tuple of signs. Let $\chi$ be the character corresponding to the $m$-tuple $(-,-,+, \ldots,+)$. We extend $\chi$ to $\mathbb{Q}_{2^{m}}^{\times}$so that it is trivial on the first two factors. Since $W_{\mathbb{Q}_{2} m}^{a b} \cong \mathbb{Q}_{2^{m}}^{\times}$we can view $\chi$ as a character of $W(K)$. Define

$$
\phi_{2}=\operatorname{Ind}_{W_{\mathbb{Q}_{2} m}}^{W_{\mathbb{Q}_{2}}}(\chi) .
$$

Since the conjugates $\chi \circ \operatorname{Fr}_{2}^{i}$, for $i=1, \ldots, m$, are mutually distinct this representation is irreducible by Mackey's criterion. Since $\chi$ is quadratic the representation $\phi_{2}$ is also delf-dual and, since $m$ is odd, it is orthogonal. Thus $\phi_{2}$ defines a self-dual supercuspidal representation $\Pi_{2}$ of $\mathrm{GL}_{2 n+1}\left(\mathbb{Q}_{2}\right)$ by the local Langlands correspondence.

For later purposes we need to describe the image of the representation $\phi_{2}$. Note that the intersection of the kernels of $\chi \circ \operatorname{Fr}_{2}^{i}$ is equal to $\Delta \mathbb{F}_{2}$, the diagonal in $\mathbb{F}_{2}^{m}$.

Proposition 4.1. Recall that $m=2 n+1$. Let $I$ be the image of $W\left(\mathbb{Q}_{2}\right)$ under the representation $\phi_{2}$. Then

(1) $I /[I, I] \cong \mathbb{Z} / m \mathbb{Z}$.

(2) $[I, I] \cong \mathbb{F}_{2}^{m} / \Delta\left(\mathbb{F}_{2}\right)$.

(3) I is contained in a special orthogonal group.

(4) If $m=7$ then $I$ is not contained in $\mathrm{G}_{2}$.

Proof. Since $W_{\mathbb{Q}_{2}} / W_{\mathbb{Q}_{2} m}$ is a cyclic group of order $m$, in order to prove the first two statements, it suffices to show that the commutator is given by the image of $W_{\mathbb{Q}_{2} m}$. Note that the commutator of $e_{i}$ and $\mathrm{Fr}_{2}$ in $W_{\mathbb{Q}_{2}}$ is equal to $e_{i}+e_{i+1}$, considered as an element of $U / U_{1} \cong \mathbb{F}_{2}^{m}$. Since $m$ is odd, these elements generate $\mathbb{F}_{2}^{m} / \Delta\left(\mathbb{F}_{2}\right)$. The first two statements 
now follow. Since the determinant character is of order two and $I /[I, I]$ is odd, it has to be trivial on $I$. This shows the third statement. Finally, if $m=7$, then $\phi_{2}\left(e_{i}\right)$ has eigenvalues 1 (with multiplicity 5) and -1 (with multiplicity 2). These cannot be arranged as a family of type $\lambda_{1}^{ \pm 1}, \lambda_{2}^{ \pm}, \lambda_{3}^{ \pm}$and 1 such that $\lambda_{1} \lambda_{2} \lambda_{3}=1$. The proposition is proved.

We now consider the Jordan subgroup in $G_{2}$. the intermediate group $J \subseteq I \subseteq N$ in advance so that $I / J$ is the normalizer of an elliptic torus in $N / J \cong \mathrm{SL}_{3}(2)$. In particular, if we identify $J$ with $\mathbb{F}_{2^{3}}$ then $I / J$ can be identified as a semi-direct product of $\operatorname{Gal}\left(\mathbb{F}_{2^{3}} / \mathbb{F}_{2}\right)$ and $\mathbb{F}_{2^{3}}^{\times}$. Since the order of $I / J$ is prime to $J$ one easily check that this extension splits. Note that $I / J$ acts transitively on the set of non-trivial irreducible characters of $J$. Let $\psi$ be a non-trivial additive character of $\mathbb{F}_{2}$. Then the composition of the trace $\operatorname{Tr}: \mathbb{F}_{2^{3}} \rightarrow \mathbb{F}_{2}$ is a character of $\mathbb{F}_{2^{3}}$ such that its stabilizer in $I / J$ is $\operatorname{Gal}\left(\mathbb{F}_{2^{3}} / \mathbb{F}_{2}\right)$. It follows, from Mackey's theory, that $I$ has 3 irreducible faithful representations, of dimension 7 , only one of which is self-dual.

Proposition 4.2. Let $\varphi: W_{\mathbb{Q}_{2}} \rightarrow \mathrm{G}_{2}$ be a parameter with the image I. Let $\phi_{2}: W_{\mathbb{Q}_{2}} \rightarrow$ $\mathrm{GL}_{7}(\mathbb{C})$ obtained by natural inclusion $\mathrm{G}_{2} \subseteq \mathrm{GL}_{7}(\mathbb{C})$. Then $\phi_{2}$ is a self-dual, irreducible representation of $W_{\mathbb{Q}_{2}}$.

Proof. This is easy. We know that any irreducible representation of $I$ either has $J$ in the kernel or it is faithful, in which case it is of the dimension $2^{3}-1=7$. There are three such representations, only one is is self-dual. Since the restriction of the standard representation of $\mathrm{G}_{2}$ to $I$ is faithful and self-dual, it must be isomorphic to the unique irreducible self-dual representation of $I$ of the same dimension.

It remains to show that the group $I$ can be obtained as the image of the Weil group $W_{\mathbb{Q}_{2}}$. Let $L$ be the Galois extension of $\mathbb{Q}_{2}$ given as the totally ramified extension of $\mathbb{Q}_{2^{3}}$ of degree 7. In other words, $L$ is the splitting field of the polynomial

$$
X^{7}-2=0
$$

Note that the Galois group of $L$ is isomorphic to $I / J$. Let $\varpi$ be a uniformizer in $L, U \subseteq L^{\times}$ the maximal pro-2 subgroup and $U \supseteq U_{1} \supseteq \ldots$ the usual filtration. Then

$$
L^{\times}=\langle\varpi\rangle \times \mathbb{F}_{2^{3}}^{\times} \times U .
$$

Let $\chi$ be a character of $W_{L}^{a b} \cong L^{\times}$which is trivial on the the first two factors of $L^{\times}$and a non-trivial character of $U / U_{1} \cong \mathbb{F}_{2^{3}}$. Consider the induced representation

$$
\operatorname{Ind}_{W_{L}}^{W_{\mathbb{Q}_{2}}}(\chi)
$$

This representation breaks up as a sum of three irreducible representations of dimension 7 , one of which is self-dual. $W_{K}$ be the kernel of this representation. Then the Galois group of $K$ over $\mathbb{Q}_{2}$ is isomorphic to $I$. In other words, we have constructed map $\varphi: W_{\mathbb{Q}_{2}} \rightarrow G^{*}$ with the image $I$. 


\section{LOCAL Lift FROM $\mathrm{G}_{2}$ TO $\mathrm{PGSp}_{6}$}

The dual group of $\mathrm{PGSp}_{6}\left(\mathbb{Q}_{p}\right)$ is $\operatorname{Spin}_{7}(\mathbb{C})$. The group $\operatorname{Spin}_{7}(\mathbb{C})$ has a unique open orbit on the 8-dimensional spin representation. The stabilizer of a point in the open orbit is isomorphic to $\mathrm{G}_{2}(\mathbb{C})$. This gives an embedding

$$
f: \mathrm{G}_{2}(\mathbb{C}) \rightarrow \operatorname{Spin}_{7}(\mathbb{C})
$$

of dual groups, indicating that there should be a functorial, but non-endoscopic, lift of representations from $\mathrm{G}_{2}\left(\mathbb{Q}_{p}\right)$ to $\mathrm{PGSp}_{6}\left(\mathbb{Q}_{p}\right)$, once local Langlands parametrizations for the two groups are established. The Langlands parameterization is essentially known for depth zero representations. We shall now spell out some special cases of our interest. In order to simplify notation let

$$
\left\{\begin{array}{l}
G=\mathrm{G}_{2}\left(\mathbb{Q}_{p}\right) \\
G^{\prime}=\mathrm{PGSp}_{6}\left(\mathbb{Q}_{p}\right) .
\end{array}\right.
$$

Recall that a root of one $\tau$ such that $\tau^{p^{2}-p+1}=1$ defines a 7 -dimensional orthogonal parameter $\phi(\tau) \oplus \chi_{p}$ which is contained in $\mathrm{G}_{2}(\mathbb{C})$. Therefore, it defines a generic supercuspidal representation denoted by $\sigma(\tau)$ of $G$ and, by composing this parameter with the inclusion $f$, a generic supercuspidal representation $\sigma^{\prime}(\tau)$ of $G^{\prime}$. The representation $\sigma^{\prime}(\tau)$, when restricted to $\operatorname{Sp}_{6}\left(\mathbb{Q}_{p}\right)$, breaks up as a sum of two representations in the $L$-packet for the parameter $\phi(\tau) \oplus \chi_{p}$.

We have the following:

- The functorial lift of the supercuspidal representation $\sigma(\tau)$ is the supercuspidal representation $\sigma^{\prime}(\tau)$.

- The functorial lift of the Steinberg representation $\mathrm{st}_{G}$ is the Steinberg representation $\mathrm{st}_{G^{\prime}}$.

- Let $\sigma$ be an unramified representation $\sigma$ of $G$ corresponding to a semi-simple conjugacy class (Satake parameter) $s \in \mathrm{G}_{2}(\mathbb{C})$. Then the lift of $\sigma$ is $\sigma^{\prime}$, an unramified representation of $G^{\prime}$ corresponding to the parameter $s^{\prime}=f(s)$.

Although the local parametrizations for $G$ and $G^{\prime}$ are not complete, a lift from $G$ to $G^{\prime}$ is given by a correspondence arising from the minimal representation $\Sigma$ of the split, adjoint $\mathrm{E}_{7}\left(\mathbb{Q}_{p}\right)$. More precisely, if $\sigma$ is an irreducible representation of $G$, then we define $\Theta(\sigma)$ to be the set of isomorphism classes of all irreducible representations $\sigma^{\prime}$ of $G^{\prime}$ such that $\sigma \otimes \sigma^{\prime}$ is a quotient of $\Sigma$.

Let $\psi: U \rightarrow \mathbb{C}^{\times}$be a Whittaker character for $G$, where $U$ is a maximal unipotent subgroup of $G$. Recall that a representation $\sigma$ of $G$ is $\psi$-generic (or simply generic) if $\sigma_{U, \psi}$, the space of $\psi$-twisted $U$-coinvariants, is nonzero. We have the same definition for representations of $G^{\prime}$ with respect to a Whittaker character $\psi^{\prime}: U^{\prime} \rightarrow \mathbb{C}^{\times}$, where $U^{\prime}$ is a maximal unipotent subgroup of $G^{\prime}$. Let $\Theta_{\text {gen }}(\sigma)$ be the subset of $\Theta(\sigma)$ consisting of generic representations. This set is somewhat easier to determine.

Proposition 5.1. Let $\sigma$ be an irreducible representation of $G$. Then $\Theta_{\operatorname{gen}}(\sigma) \neq 0$ only if $\sigma$ is generic. Moreover, $\Theta_{\text {gen }}(\sigma)$ contains at most one element. 
Proof. Let $\sigma^{\prime}$ be in $\Theta_{g e n}(\sigma)$. Then $\sigma \otimes \sigma^{\prime}$ is a quotient of $\Sigma$. Since $\sigma_{U^{\prime}, \psi^{\prime}}^{\prime}$ is one dimensional, we see that $\sigma$ is a quotient of $\Sigma_{U^{\prime}, \psi^{\prime}}$. Since ([Ga, Theorem 7.1])

$$
\Sigma_{U^{\prime}, \psi^{\prime}}=\operatorname{ind}_{U}^{G}(\psi)
$$

as a $G$-module, it follows that $\sigma$ is indeed generic. Moreover, since

$$
\operatorname{Hom}_{G}\left(\operatorname{ind}_{U}^{G}(\psi), \sigma\right)
$$

is one-dimensional for any generic representation $\sigma$ (by uniqueness of the Whittaker functional), the second part follows immediately. The proposition is proved.

Given a generic representation $\sigma$, the above proposition allows us to show that $\Theta_{\text {gen }}(\sigma)=$ $\left\{\sigma^{\prime}\right\}$ by simply showing that $\sigma \otimes \sigma^{\prime}$ is a quotient of $\Sigma$.

Proposition 5.2. Assume that $\sigma$ is an irreducible representation of $G=\mathrm{G}_{2}\left(\mathbb{Q}_{p}\right)$, belonging to either of the following two families:

(1) Supercuspidal representations $\sigma(\tau)$.

(2) Generic unramified representations.

Then $\Theta_{\text {gen }}(\sigma) \neq \emptyset$ and the unique representation in $\Theta_{\text {gen }}(\sigma)$ is the functorial lift of $\sigma$, as described above.

Proof. The supercuspidal representation $\sigma(\tau)$ is induced from a cuspidal representation $\rho$ of the finite group $\mathrm{G}_{2}\left(\mathbb{F}_{p}\right)$, inflated to a hyperspecial maximal compact subgroup of $G$. Similarly, $\sigma^{\prime}(\tau)$ is induced from a cuspidal representation $\rho^{\prime}$ of $\mathrm{PGSp}_{6}\left(\mathbb{F}_{p}\right)$. Gan shows in Ga that $\rho \otimes \rho^{\prime}$ is a summand of the minimal representation of the adjoint group $\mathrm{E}_{7}\left(\mathbb{F}_{p}\right)$. By $[\mathrm{Sa}$ ] the minimal representation of $\mathrm{E}_{7}\left(\mathbb{F}_{p}\right)$ appears as the first non-trivial $K$-type of the minimal representation of $\mathrm{E}_{7}\left(\mathbb{Q}_{p}\right)$. (Here $K$ is a hyperspecial maximal compact subgroup in $\mathrm{E}_{7}\left(\mathbb{Q}_{p}\right)$. In particular, $\mathrm{E}_{7}\left(\mathbb{F}_{p}\right)$ is a quotient of $K$ by the first congruence subgroup, and the $K$-type is obtained by inflating the minimal representation of $\mathrm{E}_{7}\left(\mathbb{F}_{p}\right)$ to $K$.) It follows, by Frobenius reciprocity, that $\sigma(\tau) \otimes \sigma^{\prime}(\tau)$ is a summand of $\Sigma$. This shows that $\Theta_{\operatorname{gen}}(\sigma(\tau))=\left\{\sigma^{\prime}(\tau)\right\}$ as desired.

Finally, assume that $\sigma$ is an unramified representation. Let $B=T U$ be a Borel subgroup of $G$. Then $\sigma$ is a subquotient of $\operatorname{Ind}_{B}^{G}(\chi)$ for some unramified character $\chi$ of $T$. (The induction is normalized here.) Recall that any root $\alpha$ defines a co-root homomorphism $t \mapsto h_{\alpha}(t)$ from $\mathbb{Q}_{p}^{\times}$into $T$. Let $\alpha_{1}, \alpha_{2}, \alpha_{3}$ be three short roots for $G$ such that

$$
\alpha_{1}+\alpha_{2}+\alpha_{3}=0 \text {. }
$$

This choice is unique up to the action of the Weyl group of $\mathrm{G}_{2}$. We can now compose $\chi$ with the co-root homomophisms for $\alpha_{i}$. In this way we get 3 characters $\chi_{1}, \chi_{2}, \chi_{3}$ of $\mathbb{Q}_{p}^{\times}$such that $\chi_{1} \chi_{2} \chi_{3}=1$. Now, if $\sigma$ is generic then (and only then) the whole induced representation is irreducible. According to a result of Muić ([M] Proposition 3.1) this happens if and only if

$$
\chi_{i} \neq|\cdot|^{ \pm 1} \text { and } \chi_{i} / \chi_{j} \neq|\cdot|^{ \pm 1}, 1 \leq i<j \leq 3 .
$$

Next, consider the representation $\pi=\chi_{1} \times \chi_{2} \times \chi_{3}$ of $\mathrm{GL}_{3}\left(\mathbb{Q}_{p}\right)$ (here we use the notation of Bernstein and Zelevinski). Let $P=M N$ be a maximal parabolic of $G^{\prime}$ such that $M \cong$ $\mathrm{GL}_{3}\left(\mathbb{Q}_{p}\right)$ (see $\left.\mathrm{MaS}\right]$ ). Then the local lift of $\sigma$ is the unique unramified quotient $\sigma^{\prime}$ of the representation of $G^{\prime}$ obtained by inducing $\pi$. By a result of Tadic ([Ta] Theorem 7.1) this induced representation is irreducible if and only if the same conditions as those of Muic are 
satisfied. In other words, an unramified representation $\sigma$ is generic if and only if its local lift $\sigma^{\prime}$ is, and both are equal to a fully induced principal series representation. In particular, $\sigma^{\prime}=\operatorname{Ind}_{P}^{G^{\prime}}(\pi)$ (normalized induction). By Frobenius reciprocity, we have

$$
\operatorname{Hom}_{G \times G^{\prime}}\left(\Sigma, \sigma \otimes \sigma^{\prime}\right)=\operatorname{Hom}_{G \times M}\left(\Sigma_{N}, \sigma \otimes \pi\right)
$$

where $\Sigma_{N}$ is the (normalized) Jacquet functor. Next, we recall that the minimal representation of $\mathrm{E}_{6}\left(\mathbb{Q}_{p}\right)$ is a quotient of $\Sigma_{N}$ MaS and that $\sigma \otimes \pi$ is a quotient of the minimal representation of $\mathrm{E}_{6}\left(\mathbb{Q}_{p}\right)$ GaS2]. It follows that $\operatorname{Hom}_{G \times M}\left(\Sigma_{N}, \sigma \otimes \pi\right) \neq 0$ and $\sigma \otimes \sigma^{\prime}$ is a quotient of $\Sigma$, as desired.

\section{Global FORMS}

Recall that $G$ is the split $\mathrm{Sp}_{2 n}$ or $\mathrm{G}_{2}$ over $\mathbb{Q}$. Fix a prime $\ell$ and $q$ an odd prime different from $\ell$. By Theorem 4.5 [KLS] there exists a globally generic cuspidal automorphic representation $\sigma$ of $G(\mathbb{A})$ such that

- $\sigma_{\infty}$ is a generic integrable discrete series representation.

- $\sigma_{q}$ is a tame supercuspidal generic representation; $\sigma_{q}=\sigma(\tau)$ if $G=\mathrm{G}_{2}$.

- $\sigma_{v}$ is unramified for all $v \neq 2, q, \ell$.

- If $G=\mathrm{G}_{2}$ then $\sigma_{2}$ is unramified, and if $G=\mathrm{Sp}_{2 n}, \sigma_{2}$ is the Jiang-Soudry descent of the self-dual supercuspidal representation of $\Pi_{2}$ introduced in Section 4 .

The form $\sigma$ lifts to an irreducible, self-dual, automorphic representation $G_{2 n+1}(\mathbb{A})$ or $G_{2}(\mathbb{A})$, with trivial central character. This uses the lift of Cogdell et al if $G$ is $\operatorname{Sp}_{2 n}$. If $G$ is $\mathrm{G}_{2}$ we first use the exceptional theta lift GRS to obtain a non-zero generic automorphic form $\sigma^{\prime}$ on $\operatorname{PGSp}_{6}(\mathbb{A})$. The form $\sigma^{\prime}$ is cuspidal if the lift of $\sigma$ to $\operatorname{PGL}_{3}(\mathbb{A})$ (via the minimal representation of $\left.E_{6}\right)$ is 0 . This holds since $\sigma_{\infty}$ is a discrete series representation and it cannot appear as a local component in the lift from $\mathrm{PGL}_{3}(\mathbb{A})$ GaS1. Thus, $\sigma^{\prime}$ is a generic cuspidal automorphic representation and its local $p$-adic components are determined by Proposition 5.2. The infinitesimal character of the real component $\sigma_{\infty}^{\prime}$ is integral and regular by the matching of infinitesimal characters in [HPS]. Next, we restrict $\sigma^{\prime}$ to $\operatorname{Sp}_{6}(\mathbb{A})$ and use the lift of Cogdell at al to obtain an automorphic representation $\Pi$ of $\mathrm{GL}_{7}(\mathbb{A})$.

Recall that $\chi_{q}$ is the unique non-trivial quadratic unramified character of the local Weil group. The local components of $\Pi$ satisfy:

- $\Pi_{\infty}$ has a regular and integral infinitesimal character.

- $\Pi_{q}$ has the parameter $\phi(\tau) \oplus \chi_{q}$.

- $\Pi_{v}$ is unramified for all $v \neq 2, q, \ell$.

- If $\ell \neq 2$ then $\Pi_{2}$ is unramified or, if $G=\mathrm{Sp}_{2 n}$, it has the irreducible parameter $\phi_{2}$.

Note that if $\Pi_{v}$ is unramified than the eigenvalues of its Satake parameter are

$$
\lambda_{1}^{ \pm 1}, \ldots, \lambda_{n}^{ \pm 1}, 1
$$

Moreover, if $G$ is $\mathrm{G}_{2}$ then we have one additional relation:

$$
\lambda_{1} \lambda_{2} \lambda_{3}=1
$$

If $\Pi_{2}$ is the self-dual supercuspidal representation of $\mathrm{GL}_{2 n+1}\left(\mathbb{Q}_{2}\right)$ with the parameter $\phi_{2}$ then the lift $\Pi$ is clearly supercuspidal. If $\Pi_{2}$ is unramified then, since the parameter of $\sigma_{q}$ is not irreducible, the global representation $\Pi$ might not be cuspidal. We give a criterion which 
guarantees that it is. Note that the conditions of the following proposition are automatically satisfied if $q$ and the parameter $\phi(\tau) \oplus \chi_{q}$ are picked using Lemma 3.3 .

Proposition 6.1. Assume that $\sigma$ is a globally generic cuspidal automorphic representation of $\operatorname{Sp}_{2 n}(\mathbb{A})$, such that $\sigma_{v}$ is unramified at all (finite) primes $v \neq \ell, q$ and $\sigma_{q}$ corresponds to the parameter $\phi(\tau) \oplus \chi_{q}$. If $q$ splits in all quadratic extensions of $\mathbb{Q}$ ramified at $\ell$ and no other primes then $\Pi$, the global lift of $\sigma$ to $\mathrm{GL}_{2 n+1}(\mathbb{A})$, is cuspidal.

Proof. If $\Pi$ is not cuspidal then by [CKPS] and forced by the local parameter of $\Pi_{q}$ we have an isobaric sum

$$
\Pi=\Sigma \boxplus \chi
$$

where $\Sigma$ is a cuspidal self-dual automorphic representation of $G_{2 n}(\mathbb{A})$ and $\chi$ is a quadratic character of $\mathrm{GL}_{1}(\mathbb{A})$. The local component $\chi_{v}$ of $\chi$ is clearly unramified for all primes $v \neq \ell, q$. It is also unramified and non-trivial at $q$ since it corresponds, via the local class field theory, to the one-dimensional summand of the parameter of $\Pi_{q}$ (denoted by the same symbol $\chi_{q}$ ). By the global class field theory $\chi$ corresponds to a quadratic extension of $\mathbb{Q}$ ramified at $\ell$ and no other primes, and such that $q$ is inert. This is a contradiction.

\section{Reductive Groups}

Let $G$ be a connected reductive group over an algebraically closed field of characteristic different from 2, and let $T$ be a maximal torus in $G$. A representation $V$ of $G$ is called almost miniscule if $V^{T} \neq 0$ and the Weyl group acts transitively on the set of non-trivial weights of $V$. These representations can be easily classified for an almost simple $G$. Assume first that the field characteristic is 0 . Since $V^{T} \neq 0$ then, by Lie algebra action, $V$ contains a root as a weight. All non-zero weights are now Weyl-group conjugates of that root. Therefore, if $G$ is simply laced then $V$ is the adjoint representation of $G$. If $G$ is multiply laced then the weights are all short roots. We tabulate possible cases:

\begin{tabular}{|c|c|c|}
\hline$G$ & $\operatorname{dim}(V)$ & $\operatorname{dim}\left(V^{T}\right)$ \\
\hline $\mathrm{B}_{n}$ & $2 n+1$ & 1 \\
$\mathrm{C}_{n}$ & $2 n^{2}-n-1$ & $n-1$ \\
$\mathrm{G}_{2}$ & 7 & 1 \\
$\mathrm{~F}_{4}$ & 26 & 2 \\
\hline
\end{tabular}

It is interesting to note that dimension of $V^{T}$ is equal to the number of short simple roots. The Weyl group acts, naturally, on $V^{T}$. The action of long root reflections is trivial, while $V^{T}$ is a reflection representation for the subgroup generated by simple short root reflections.

Proposition 7.1. Let $G$ be an almost simple group over an algebraically closed field of characteristic $\ell>2$. Then:

(1) Any miniscule representation is isomorphic to a Frobenius twist of a representation with a miniscule weight as the highest weight.

(2) Assume first that $\ell \neq 3$ if $G=\mathrm{G}_{2}$. Then any almost miniscule representation is a Frobenius twist of the almost miniscule representation with the highest weight equal to a short root. If $\ell=3$ and $G=\mathrm{G}_{2}$ then there is one additional family of miniscule representations. It consists of Frobenius twists of the representation with the highest weight equal to a long root. 
Proof. Let $\alpha_{1}, \ldots, \alpha_{r}$ be the simple roots for $G$. We shall characterize miniscule (and then almost miniscule) representations with the highest weight $\lambda$ such that $0 \leq\left\langle\lambda, \alpha_{i}^{\vee}\right\rangle \leq \ell-1$ for all $i$. The general case can be easily deduced from the Steinberg tensor product theorem.

For any root $\alpha$, the group $G$ contains a subgroup isomorphic to (a quotient of) $\mathrm{SL}_{2}$. If $\left\langle\lambda, \alpha^{\vee}\right\rangle=n \leq \ell-1$ then the action of $\mathrm{SL}_{2}$ on the highest weight vector will give rise to the weights $\lambda, \lambda-\alpha, \ldots \lambda-n \alpha$. By examining the lengths of these weights we see that only the last one is in the Weyl group orbit of $\lambda$. This forces $n=0$ or 1 if the representation is to be miniscule. In particular, if we write $n_{i}=\left\langle\lambda, \alpha_{i}^{\vee}\right\rangle$, then $n_{i}=0$ or 1 for all $i$. Next, we claim that only one $n_{i}$ could be 1 . Otherwise, we can pick a path in the Dynkin diagram $\alpha_{i}, \alpha_{i+1}, \ldots, \alpha_{j}$ such that $n_{i}=n_{j}=1$ and $n_{k}=0$ for any $\alpha_{k}$ between $\alpha_{i}$ and $\alpha_{j}$. Consider the root $\alpha=\alpha_{i}+\alpha_{i+1}+\cdots+\alpha_{j}$. Since $\left\langle\lambda, \alpha^{\vee}\right\rangle=2$, this is a contradiction. (Note that we have just used the condition $\ell \neq 2$.) Finally, if the weight $\lambda$ is fundamental but not miniscule then there exists a positive root $\alpha$ such that $\left\langle\lambda, \alpha^{\vee}\right\rangle=2$. Again, a contradiction.

The proof of (2) is similar, except when $\left\langle\lambda, \alpha^{\vee}\right\rangle=2$. Then $\lambda, \lambda-\alpha$ and $\lambda-2 \alpha$ are weights with $\lambda-\alpha$ the shortest length among the three weights. Since 0 is the only other orbit of weights, we must have $\lambda=\alpha$. If $\alpha$ is a long highest root, and the root system is of the type $B_{n}, C_{n}$ or $F_{4}$ then there exists a short root $\beta$ such that $\left\langle\alpha, \beta^{\vee}\right\rangle=2$. This implies that the short root $\alpha-\beta$ is also a weight, and the representation cannot be almost miniscule. If $\ell=3$ and $G=\mathrm{G}_{2}$ then this argument breaks down. The adjoint representation breaks up as $14=7+7^{\prime}$ where 7 is the representation whose non-trivial weights are short roots, while $7^{\prime}$ is a representation whose non-trivial weights are long roots.

We also note that the 26-dimensional almost miniscule representation of $\mathrm{F}_{4}$ is not irreducible. It breaks up as $26=25+1$. The main result of this section is the following:

Proposition 7.2. Let $G$ be a connected reductive group over an algebraically closed field of characteristic different from 2. Let $V$ be an irreducible and faithful representation of $G$ of dimension $2 n+1$, preserving a non-degenerate bilinear form. Assume that there exist $2 n$ different weights in $V$ permuted cyclically by a Weyl group element. Then $G=\mathrm{SO}_{2 n+1}$ or $\mathrm{G}_{2}$ if $n=3$.

Proof. Let $Z$ be the connected component of the center of $G$. The characters of $Z$ form a lattice. In particular, the only self-dual character is the trivial character. This shows, since $V$ is faithful, that $Z$ is trivial. Therefore $G$ is semi-simple. Since weights of a self-dual representation come in pairs $\{\mu,-\mu\}$, and the Weyl group preserves the length of weights, the weight outside the cycle must be trivial, so $\operatorname{dim}\left(V^{T}\right)=1$. Next, let $G_{1} \times \cdots \times G_{k}$ be a product of almost simple groups isogenous to $G$ such that $V=V_{1} \otimes \cdots \otimes V_{k}$ is a tensor product of irreducible, non-trivial representations of $G_{1}, \ldots, G_{k}$. Since $V^{T} \neq 0$, the zero weight must appear in each $V_{1}, \ldots, V_{k}$. But then $V$ can be almost miniscule only if $k=1$. From the list of almost miniscule representations of almost simple groups we see that $V$ must be the standard representation of $\mathrm{SO}_{2 n+1}$ or $G_{2}$ or a Frobenius twist of it, if $\ell \neq 3$. If $\ell=3$ then $\mathrm{F}_{4}$ has an almost miniscule representation of dimension 25 . However, since $\mathrm{F}_{4}$ has no element of order 24 in its Weyl group, there is no element permuting all non-trivial weights. This completes the proof. 


\section{Galois Representation}

Let $\Pi$ be the self-dual cuspidal automorphic representation of $\mathrm{GL}_{2 n+1}(\mathbb{A})$ constructed by lifting from $\mathrm{Sp}_{2 n}$, or from $\mathrm{G}_{2}(\mathbb{A})$ in Section 6 . In particular,

- The infinitesimal character of $\Pi_{\infty}$ is regular and integral.

- The local component $\Pi_{v}$ is unramified for all $v \neq \ell, q$

- The local parameter of $\Pi_{q}$ is $\phi(\tau) \oplus \chi_{q}$ where $q$ splits in any quadratic extension of $\mathbb{Q}$ ramified at $\ell$ only.

- If $\ell \neq 2$ then $\Pi_{2}$ is unramified or, if $G=\mathrm{Sp}_{2 n}$, the local parameter is the irreducible representation $\phi_{2}$.

By the generalization of Theorem 1.1 due to Shin, and the remark after it, one can attach a semi-simple Galois representation to $\Pi$ :

$$
r_{\Pi}: G_{\mathbb{Q}} \rightarrow \mathrm{GL}_{2 n+1}\left(\overline{\mathbb{Q}}_{\ell}\right)
$$

such that for every prime $v \neq \ell$ the restriction of $r$ to the decomposition group $D_{v}$ gives the Langlands parameter of $\Pi_{v}$, up to a Frobenius semi-simplification.

[Note that when the Weil-Deligne parameter $\mathcal{L}\left(\Pi_{v}\right)$ for all $v \neq \ell$ has monodromy $N=0$, we can state Theorem 1.1 as :

$$
r_{\Pi}||_{D_{v}}^{\text {Frob-ss }}=\mathcal{L}\left(\Pi_{v}\right)
$$

and where $\mathcal{L}\left(\Pi_{v}\right)$ may be regarded, using the embedding $\iota: \overline{\mathbb{Q}} \hookrightarrow \overline{\mathbb{Q}}_{\ell}$, as a representation of the decomposition group $D_{v}$ at $v$ with values in $\mathrm{GL}_{2 n+1}\left(\overline{\mathbb{Q}}_{\ell}\right)$.]

Let us concentrate on the prime $q$. Here $\Pi_{q}$ is the lift of a supercuspidal representation of $\operatorname{Sp}_{2 n}\left(\mathbb{Q}_{q}\right)$ whose parameter, when restricted to the inertia group $I_{\mathbb{Q}_{q}}$, is a direct sum of $2 n+1$ one dimensional characters. One trivial and $2 n$ other cyclically permuted by $\operatorname{Fr}_{q}$. It follows that $r_{\Pi}\left(\operatorname{Fr}_{q}^{2 n}\right)$ commutes with $r_{\Pi}\left(I_{q}\right)$. This shows that $r_{\Pi}\left(\operatorname{Fr}_{q}^{2 n}\right)$ and $r_{\Pi}\left(\operatorname{Fr}_{q}\right)$ must be semi-simple. In other words, $r$ gives exactly the parameter of $\Pi_{q}$. The same argument shows that the restriction of $r_{\Pi}$ to $D_{2}$ gives the parameter of $\Pi_{2}$ if $\Pi_{2}$ is supercuspidal.

Proposition 8.1. If $\Pi$ satisfies the above conditions at places $\infty, 2$ and $q$ then the Galois representation $r_{\Pi}$ is irreducible and orthogonal.

Proof. If $\Pi_{2}$ is supercuspidal, then the local parameter is irreducible and $r_{\Pi}$ is irreducible. Thus, assume that $\Pi_{2}$ is unramified (or $\ell=2$ ). If $r_{\Pi}$ is reducible then $r_{\Pi}$ has two irreducible summands of dimensions $2 n$ and 1 . Since the eigenvalues of $r_{\Pi}\left(\operatorname{Fr}_{v}\right)$ are $1, \lambda_{i}^{ \pm},(1 \leq i \leq n)$ the representation $r_{\Pi}$ is self-dual. In particular, the one-dimensional summand is a quadratic character $\chi$ ramified at $\ell$ only and such that $\chi\left(\mathrm{Fr}_{q}\right)=-1$. This implies that there exists a quadratic extension ramified at $\ell$ only and such that $q$ stays inert. This is a contradiction since $q$ is picked so that it splits completely in every quadratic extension ramified at $\ell$ only. Therefore $r_{\Pi}$ is irreducible. Since it is self-dual and of odd dimension it is orthogonal as well.

\section{ZARISKI CLOSURE}

We shall now assume that the odd prime $q$ and the parameter $\phi(\tau) \oplus \chi_{q}$ of the local component $\Pi_{q}$ of $\Pi$ are picked using Lemma 3.3. In particular, the representation $r_{\Pi}$ is unramified at all primes different from $2, \ell$ and $q$, and $r_{\Pi}\left(I_{q}\right)$, the image of the inertia 
subgroup is of order $p$. Let $\Gamma=r_{\Pi}\left(G_{\mathbb{Q}}\right)$. If $d>1$ is an integer, let $\Gamma^{d}$ be the intersection of all normal subgroups of $\Gamma$ of index $\leq d$. The following is crucial:

Lemma 9.1. Assume that the local component $\Pi_{q}$ is constructed by means of Lemma 3.3. Then $r_{\Pi}\left(D_{q}\right)$ is contained in $\Gamma^{d}$.

Proof. Let $\Gamma^{\prime}$ be a normal subgroup of index $\leq d$. We must show that the image of $D_{q}$ lands in $\Gamma^{\prime}$. Let $L$ be the Galois extension of $\mathbb{Q}$ corresponding to $\Gamma^{\prime}$. Obviously, $L$ is unramified at all primes except $2, \ell$ and $q$. Moreover, since $r_{\Pi}\left(I_{q}\right)$ is of order $p$ and $p>d$, it follows that $L$ is unramified at $q$ as well. Thus, $L$ is contained in $K$, the compositum of all Galois extensions ramified at $2, \ell$ and of degree $\leq d$. This implies that $q$ splits completely in $L$ (by Lemma 3.3) and $r_{\Pi}\left(D_{q}\right)$ is therefore contained in $\Gamma^{\prime}$, as desired.

The above lemma shows that if $\Pi_{q}$ is constructed by means of Lemma 3.3 then $\Gamma_{2 n, p}$, the image of the decomposition group $D_{q}$, sits deeply embedded in $\Gamma=r_{\Pi}\left(G_{\mathbb{Q}}\right)$. This property is crucial in controling the size of the image $\Gamma$ of the Galois group.

Theorem 9.2. There exists a function $I: \mathbb{N} \rightarrow \mathbb{N}$ such that if $\Pi$ is a self-dual cuspidal automorphic representation of $\mathrm{GL}_{2 n+1}(\mathbb{A})$, as in Section 8 , and such that the local parameter of $\Pi_{q}$ is constructed by means of Lemma 3.3 with $d>I(n)$ then the Zariski closure of $r_{\Pi}\left(G_{\mathbb{Q}}\right)$ is isomorphic to $\mathrm{SO}_{2 n+1}\left(\overline{\mathbb{Q}}_{\ell}\right)$ if $n \neq 3$ and $\mathrm{SO}_{7}\left(\overline{\mathbb{Q}}_{\ell}\right)$ or $\mathrm{G}_{2}\left(\overline{\mathbb{Q}}_{\ell}\right)$ if $n=3$.

Proof. Let $G$ be the Zariski closure of $r_{\Pi}\left(G_{\mathbb{Q}}\right)$. Since $r_{\Pi}$ is irreducible this is a reductive group. Let $G^{\circ}$ be its connected component. Recall that the image of the inertia subgroup $I_{q}$ contains an element $s$ in $\mathrm{GL}_{2 n+1}\left(\overline{\mathbb{Q}}_{\ell}\right)$ of order $p$. We want to show that $s$ is contained in $G^{\circ}$. We need the following (Lemma 6.3 in [KLS] $)$.

Lemma 9.3. There exists a function $J: \mathbb{N} \rightarrow \mathbb{N}$ such that every integer $n>0$ and every algebraic subgroup $G \subset \mathrm{GL}_{n}$ over a field of characteristic zero, there is normal subgroup $G_{1} \subseteq G$ of index $\leq J(n)$ containing $G^{\circ}$ such that $G_{1} / G^{\circ}$ is abelian.

If we pick $d>J(n)$ then, by Lemma 9.1, the image of $D_{q}$ must be contained in $G_{1}$. Since $\Gamma_{2 n, p}$ is a semi-direct product of $\mathbb{Z} / p \mathbb{Z}$ and $\mathbb{Z} / 2 n \mathbb{Z} \subset$ Aut $(\mathbb{Z} / p \mathbb{Z}) \cong \mathbb{Z} /(p-1) \mathbb{Z}$ one easily sees that the commutator subgroup of $r_{\Pi}\left(D_{q}\right)$ is $r_{\Pi}\left(I_{q}\right) \cong \mathbb{Z} / p \mathbb{Z}$. This shows that the projection of $r_{\Pi}\left(D_{q}\right)$ to the abelian quotient $G_{1} / G^{\circ}$ must contain $s$ in the kernel. In other words, $s$ is in $G^{\circ}$.

Recall that the eigenvalues of $s$ are

$$
\tau, \tau^{q}, \ldots, \tau^{q^{n-1}}, \tau^{-1}, \tau^{-q}, \ldots, \tau^{-q^{n-1}}, 1 .
$$

where $\tau$ is a $p$ th root of one. Moreover, these eigenvaules are distinct. It follows that the the centralizer of $s$ in $\mathrm{GL}_{2 n+1}$ is a torus. Thus, $s$ is a regular semi-simple element in $G^{\circ}$ and the centralizer of $s$ in $G^{\circ}$ is a maximal torus $T$. Next, note that the centralizer of $s$ in $G^{\circ}$ is the same as the centralizer of $r_{\Pi}\left(\mathrm{Fr}_{q}\right) \cdot s \cdot r_{\Pi}\left(\mathrm{Fr}_{q}^{-1}\right)$ (since it is so in $\mathrm{GL}_{2 n+1}$ ). It follows that $r_{\Pi}\left(\mathrm{Fr}_{q}\right)$ normalizes $T$. We need the following lemma.

Lemma 9.4. There exists a function $J^{\prime}: \mathbb{N} \rightarrow \mathbb{N}$ such that every integer $n>0$ and every reductive group $G$ of rank $n$ over an algebraically closed field of characteristic zero, there is normal subgroup $G_{1} \subseteq G$ of index $\leq J^{\prime}(n)$ containing $G^{\circ}$ such that conjugation of $G^{\circ}$ by any element in $G_{1}$ is inner. 
Proof. The conjugation action of $G$ on $G^{\circ}$ gives a homomorphism from a finite group $G / G^{\circ}$ to Aut $\left(G^{\circ}\right) / \operatorname{Inn}\left(G^{\circ}\right)$. The latter group is contained in the group of automorphisms of a quadruple $\left(X, \Delta, X^{\vee}, \Delta^{\vee}\right)$ where $X$ and $X^{\vee}$ are the character and co-character lattices of a maximal torus $T$ in $G^{\circ}$, and $\Delta$ a choice of simple roots for $G^{\circ}$. It follows that $G / G^{\circ}$ maps into $\mathrm{GL}(X) \cong \mathrm{GL}_{n}(\mathbb{Z})$. Since there is a torsion-free congruence subgroup in $\mathrm{GL}_{n}(\mathbb{Z})$, the order of any finite subgroup in $\mathrm{GL}_{n}(\mathbb{Z})$ is bounded by the index of the congruence subgroup. This proves the lemma.

Now, if we pick $d>J^{\prime}(n)$ then $r_{\Pi}\left(D_{q}\right)$ must be contained in $G_{1}$. This shows that the normalizer of the torus $T$ in $G^{\circ}$ contains an element of order $2 n$ which cyclically permutes the weights corresponding to the $2 n$ eigenvalues of $s$ different from 1 . This shows that $r_{\Pi}$, under the action of $G^{\circ}$, has at most two irreducible summands, of dimension $2 n$ and 1 . In fact, since $G^{\circ}$ is a normal subgroup and $G$ acts irreducibly, a simple argument shows that $G^{\circ}$ must act irreducibly as well. It follows that $G^{\circ}$ satisfies conditions of Proposition 7.2 , so it must be either $\mathrm{SO}_{2 n+1}$ or $\mathrm{G}_{2}$. It remains to show that $G=G^{\circ}$. This is easy. First, $r_{\Pi}$ is an irreducible $2 n+1$-dimensional representation of $G$ which restricts to the standard representation of $G^{\circ}$. Second, since $\mathrm{SO}_{2 n+1}$ and $\mathrm{G}_{2}$ have no outer automorphisms, every connected component of $G$ contains an element commuting with $G^{\circ}$. Combining the two, it follows that any connected component of $G$ contains a homothety by a scalar $\lambda$. On the other hand, since $r_{\Pi}\left(\operatorname{Fr}_{v}\right)$ has 1 as one of the eigenvalues for almost all primes $v$, by Čebotarev's density the function $\operatorname{det}\left(1-r_{\Pi}(g)\right)$ must be 0 for all elements $g$ in $G$. In particular, $\lambda$ must be equal to 1 and this shows that $G=G^{\circ}$. The proposition is proved with $I(n)=\max \left(J(n), J^{\prime}(n)\right)$.

According to Theorem 9.2 there are two possibilities for the Zariski closure if $n=3$. The following two corollaries give us more precise statements in this case.

Corollary 9.5. Assume that $\Pi$ comes from $\mathrm{G}_{2}(\mathbb{A})$. Then the Zariski closure of $r_{\Pi}\left(G_{\mathbb{Q}}\right)$ is $\mathrm{G}_{2}\left(\overline{\mathbb{Q}}_{\ell}\right)$.

Proof. Let $G$ be the Zariski closure. We know that $G$ is either $\mathrm{SO}_{7}$ or $\mathrm{G}_{2}$. It suffices to show that the rank of the maximal torus $T$ is at most 2. To see this, recall that the eigenvalues of $r_{\Pi}\left(\operatorname{Fr}_{v}\right)$ are $1, \lambda_{i}^{ \pm}(i=1,2,3)$ for $v \neq q, \ell$. Therefore the characteristic polynomial of $r_{\Pi}\left(\operatorname{Fr}_{v}\right)$ is $f(x)=(x-1) g(x)$ with

$$
g(x)=x^{6}+a x^{5}+b x^{4}+c x^{3}+b x^{2}+a x+1
$$

a palindromic polynomial of degree 6 . Moreover, the condition $\lambda_{1} \lambda_{2} \lambda_{3}=1$ gives one algebraic relation on the three coefficients $a, b$ and $c$. By Cebotarev's density theorem, the same holds for the characteristic polynomial of all elements in the image of $r$ and, therefore, for all elements in $T$. In particular, the dimension of $T$ is less than or equal to 2 .

Corollary 9.6. Assume that $\Pi_{2}$, the local component of $\Pi$, is the irreducible self-dual cuspidal representation of $\mathrm{GL}_{7}\left(\mathbb{Q}_{2}\right)$ introduced in Section 四. Then the Zariski closure of $r_{\Pi}$ is $\mathrm{SO}_{7}\left(\overline{\mathbb{Q}}_{\ell}\right)$.

Proof. This is easy since the image of the inertia subgroup $I_{2}$ contains elements in $\mathrm{SO}_{7}\left(\overline{\mathbb{Q}}_{\ell}\right)$ which are not contained in $\mathrm{G}_{2}$. 


\section{A GROUP-THEORETIC CRITERION}

In this section we develop certain criteria which give us control over the image of $\ell$-adic representations in the case of exceptional groups. As such, this section is somewhat more general then what is needed for the main results in this paper. However, the results of this section might have future applications. A possibility in this direction is presented in Section 12 .

Let $\Gamma$ be a profinite group and $d \geq 2$ an integer. We define $\Gamma^{d}$ as the intersection of all open normal subgroups of $\Gamma$ of index $\leq d$.

Lemma 10.1. If $\Gamma$ is a profinite group, $\Delta$ a closed normal subgroup, and d a positive integer, then the image of $\Gamma^{d}$ in $\Gamma / \Delta$ is $(\Gamma / \Delta)^{d}$.

Proof. The image in $\Gamma / \Delta$ of every open subgroup of $\Gamma$ of index $\leq d$ is again open of index $\leq d$, and conversely, all open index $\leq d$ subgroups of $\Gamma / \Delta$ arise as images of open index $\leq d$ subgroups of $\Gamma$.

Let $n \geq 2$ be an integer and $p$ a prime congruent to $1(\bmod n)$.

Definition 10.2. By a group of type $(n, p)$, we mean any finite group $\Gamma$ with a normal subgroup $\Delta$ isomorphic to $\mathbb{Z} / p \mathbb{Z}$ such that the image of $\operatorname{Inn} \Gamma$ in Aut $\Delta$ is isomorphic to $\mathbb{Z} / n \mathbb{Z}$.

As noted in the introduction, this is slightly different from the terminology in [KLS. If $\ell \neq p$ is prime, a group of type $(n, p, \ell)$ will mean a (possibly finite) profinite group which is the extension of a group of type $(n, p)$ by a pro- $\ell$ group.

Lemma 10.3. If $0 \rightarrow \Gamma_{1} \rightarrow \Gamma_{2} \rightarrow \Gamma_{3} \rightarrow 0$ is a short exact sequence of profinite groups, $n \geq 2$, $\ell$ and $p$ are distinct primes, and $\Gamma_{1}$ is pro- $\ell$, then $\Gamma_{2}$ contains a subgroup of type $(n, p, \ell)$ if and only if $\Gamma_{3}$ does.

Proof. Any extension of a group of type $(n, p, \ell)$ by a pro- $\ell$ group is again of type $(n, p, \ell)$, so one direction is trivial.

For the other, let $\Delta_{2}$ be a closed subgroup of $\Gamma_{2}$ and $\Delta_{2}^{\prime}$ an open pro- $\ell$ subgroup of $\Delta_{2}$ such that $\Delta_{2}^{\prime \prime}:=\Delta_{2} / \Delta_{2}^{\prime}$ is of type $(n, p)$. Let $\Delta_{1}:=\Delta_{2} \cap \Gamma_{1}, \Delta_{1}^{\prime}:=\Delta_{2}^{\prime} \cap \Gamma_{1}$, and $\Delta_{1}^{\prime \prime}:=\Delta_{1} / \Delta_{1}^{\prime}$. By the snake lemma, we have a right-exact sequence

$$
\Delta_{2}^{\prime} / \Delta_{1}^{\prime} \rightarrow \Delta_{2} / \Delta_{1} \rightarrow \operatorname{coker}\left(\Delta_{1}^{\prime \prime} \rightarrow \Delta_{2}^{\prime \prime}\right) \rightarrow 0
$$

As $\Delta_{2}^{\prime}$ is pro- $\ell$, so is every quotient thereof, so $\Delta_{2} / \Delta_{1}$ is the extension of coker $\left(\Delta_{1}^{\prime \prime} \rightarrow \Delta_{2}^{\prime \prime}\right)$ by a pro- $\ell$ group.

Every quotient of a group $\Gamma_{n, p}$ of type $(n, p)$ by an $\ell$-group is again of type $(n, p)$. Indeed, the quotient map preserves the normal subgroup of $\Gamma_{n, p}$ isomorphic to $\mathbb{Z} / p \mathbb{Z}$ and therefore the image of $\operatorname{Inn} \Gamma_{n, p} \rightarrow(\mathbb{Z} / p \mathbb{Z})^{\times}$.

We remark that for the non-trivial direction, the proof uses only the fact that $\Gamma_{1}$ is the inverse limit of finite groups of prime-to- $p$ order.

Theorem 10.4. Let $\ell$ be a prime and $G$ a connected reductive algebraic group over $\overline{\mathbb{F}}_{\ell}$. There exists an absolute constant $B$ such that 
(1) If $\operatorname{rk} G \leq 2$ and $p>B$ is a prime distinct from $\ell$ and $G\left(\overline{\mathbb{F}}_{\ell}\right)$ contains a subgroup of type $(6, p, \ell)$, then $G$ is of type $\mathrm{G}_{2}$.

(2) If $\operatorname{rk} G \leq 4$ and $p_{1}, p_{2}>B$ are primes distinct from $\ell$ and $G\left(\overline{\mathbb{F}}_{\ell}\right)$ contains subgroups of type $\left(8, p_{1}, \ell\right)$ and $\left(12, p_{2}, \ell\right)$, then $G$ is of type $\mathrm{F}_{4}$.

(3) If $\operatorname{rk} G \leq 6$ and $p>B$ is a prime distinct from $\ell$ and $G\left(\overline{\mathbb{F}}_{\ell}\right)$ contains a subgroup of type $(9, p, \ell)$, then $G$ is of type $\mathrm{E}_{6}$.

(4) If $\operatorname{rk} G \leq 7$ and $p_{1}, p_{2}>B$ are primes distinct from $\ell$ and $G\left(\overline{\mathbb{F}}_{\ell}\right)$ contains subgroups of type $\left(18, p_{1}, \ell\right)$ and $\left(30, p_{2}, \ell\right)$, then $G$ is of type $\mathrm{E}_{7}$.

(5) If $\mathrm{rk} G \leq 8$ and $p_{1}, p_{2}, p_{3}>B$ are primes distinct from $\ell$ and $G\left(\overline{\mathbb{F}}_{\ell}\right)$ contains subgroups of type $\left(18, p_{1}, \ell\right),\left(20, p_{2}, \ell\right)$, and $\left(30, p_{3}, \ell\right)$, then $G$ is of type $\mathrm{E}_{8}$.

Proof. We claim that the root systems of type $\mathrm{G}_{2}, \mathrm{~F}_{4}, \mathrm{E}_{6}, \mathrm{E}_{7}$, and $\mathrm{E}_{8}$ respectively are the only root systems of rank less than or equal to $2,4,6,7$, and 8 respectively, which have Weyl group elements of order $6 ; 8$ and 12;9;18 and 30; and 18, 20, and 30 respectively. To see that this is so, we compile a table of the orders of Weyl group elements for root systems of rank $\leq 8$. We write each root system as a sum of irreducible root systems, each coded as a single letter and a single digit, and arranged alphabetically. We order root systems first by rank and within rank, alphabetically. A root system is exceptional if it is simple of type $\mathrm{G}_{2}, \mathrm{~F}_{4}, \mathrm{E}_{6}, \mathrm{E}_{7}$, or $\mathrm{E}_{8}$. For brevity, we omit those root systems for which the set of possible Weyl element orders is a proper subset of that for some non-exceptional root system of equal or inferior rank. In case of equality, we print only the lexicographically smallest example. (For example, in our table, no root system of type $C_{n}$ appears, since $B_{n}$ is lexicographically inferior to it and has the same set of Weyl group orders. Likewise, $A_{1}+A_{4}$ does not appear because its set of Weyl group orders is strictly dominated by that of $B_{5}$, which, though lexicographically superior, has the same rank.) Since the set of orders of elements of a finite group is determined by its subset of maximal elements with respect to divisibility, we exhibit only this subset.

\begin{tabular}{|c|l|}
\hline Root System & Maximal Elements \\
\hline A1 & 2 \\
\hline A2 & 2,3 \\
B2 & 4 \\
G2 & 6 \\
\hline B3 & 4,6 \\
\hline A2+B2 & 12 \\
A4 & $4,5,6$ \\
B4 & $4,6,8$ \\
F4 & 8,12 \\
\hline B5 & $8,10,12$ \\
\hline A2+B4 & 24 \\
A4+B2 & 12,20 \\
A4+G2 & 12,30 \\
A6 & $7,10,12$ \\
E6 & $8,9,10,12$ \\
\hline
\end{tabular}




\begin{tabular}{|c|l|}
\hline A1+E6 & $8,10,12,18$ \\
A2+B5 & 24,30 \\
A4+B3 & $12,20,30$ \\
A7 & $7,8,10,12,15$ \\
B7 & $14,20,24$ \\
E7 & $8,12,14,18,30$ \\
\hline A2+E6 & $18,24,30$ \\
A4+F4 & $24,40,60$ \\
A6+B2 & $12,20,28$ \\
A6+G2 & $12,30,42$ \\
A8 & $8,9,12,14,15,20$ \\
B2+E6 & $8,20,36$ \\
B8 & $14,16,20,24,30$ \\
E8 & $14,18,20,24,30$ \\
\hline
\end{tabular}

Now, let $\Gamma_{n, p, \ell}$ be a subgroup of $G\left(\overline{\mathbb{F}}_{\ell}\right)$ of type $(n, p, \ell)$ for some $n \geq 2$ and some prime $p \neq \ell$. Let $\Delta_{\ell} \subset \Gamma_{n, p, \ell}$ denote a normal $\ell$-subgroup such that the corresponding quotient group $\Gamma_{n, p}$ is of type $(n, p)$. Now, $\Delta_{\ell} \subset G\left(\mathbb{F}_{\ell^{k}}\right)$ for some $k$, and so it is contained in a Sylow $\ell$-subgroup of $G\left(\mathbb{F}_{\ell^{k}}\right)$. Such a subgroup is the group of $\mathbb{F}_{\ell^{k}}$-points of the unipotent radical of a Borel subgroup of $G$. By [Hu1, 30.3], the normalizer of $\Delta_{\ell}$ in $G$ is a parabolic subgroup $P$, proper if $\Delta_{\ell}$ is non-trivial. If $N$ denotes the unipotent radical of $P$, then $N\left(\overline{\mathbb{F}}_{\ell}\right) \cap \Gamma_{n, p, \ell}$ is an $\ell$-group, so by Lemma 10.3, the image of $\Gamma_{n, p, \ell}$ in the Levi factor $M\left(\overline{\mathbb{F}}_{\ell}\right)$ is again of type $(n, p, \ell)$. The rank of $M$ is equal to that of $G$, while the dimension is strictly less. Iterating this process we end up with a connected reductive group, which we again denote $M$, of the same rank as $G$ such that $M\left(\overline{\mathbb{F}}_{\ell}\right)$ contains a subgroup $\Gamma_{n, p}$ of type $(n, p)$. If $M$ is an exceptional group, then $G=M$, since in each rank $r$, the exceptional group, if one exists, is the connected reductive group of maximal dimension in rank $r$.

Let $\Gamma_{n, p}$ be a subgroup of type $(n, p)$ of $M\left(\overline{\mathbb{F}}_{\ell}\right)$ for some integer $n$, let $x$ denote the image of a generator of the normal subgroup of $\Gamma_{n, p}$ isomorphic to $\mathbb{Z} / p \mathbb{Z}$, and let $a \in \mathbb{Z}$ be such that its image in $\mathbb{Z} / p \mathbb{Z}$ is of order $n$ in $(\mathbb{Z} / p \mathbb{Z})^{\times}$. As $x$ and $x^{a}$ are conjugate in $\Gamma_{n, p}, x$ and $x^{a}$ are conjugate in $M\left(\overline{\mathbb{F}}_{\ell}\right)$. Since $p \neq \ell$, they are semisimple elements, and if $B<p$ is taken to be larger than the maximal number of components of the centralizer of any semisimple element in any reductive connected group of rank $\leq 8$, it follows that $x$ and $x^{a}$ belong to a common maximal torus $T \subset M$. By a well-known theorem [Hu2, $\S 3.1]$, there exists $w \in N_{M}(T)\left(\overline{\mathbb{F}}_{\ell}\right)$ such that $w x w^{-1}=x^{a}$. However, this implies that the order of the image of $w$ in the Weyl group of $M$ with respect to $T$ is divisible by $n$. From our analysis of orders of elements in Weyl groups in rank $\leq 8$, it follows that $M$ is exceptional and therefore that $G$ is exceptional.

Theorem 10.5. There exist constants $A$ and $B$ such that if

(1) $d>A$ is an integer,

(2) $p_{1}, p_{2}, p_{3}>B$ and $\ell \notin\left\{p_{1}, p_{2}, p_{3}\right\}$ are primes,

(3) $K$ is an $\ell$-adic field,

(4) $G$ is a connected reductive algebraic group over $K$ such that

(a) $\operatorname{rk} G \leq 2$,

(b) $\operatorname{rk} G \leq 4$, 
(c) $\operatorname{rk} G \leq 6$,

(d) $\operatorname{rk} G \leq 7$, or

(e) $\operatorname{rk} G \leq 8$,

(5) $\Gamma \subset G(K)$ is a profinite subgroup such that (respectively)

(a) $\Gamma^{d}$ has a subgroup of type $\left(6, p_{1}, \ell\right)$;

(b) $\Gamma^{d}$ has a subgroup of type $\left(8, p_{1}, \ell\right)$ and $\left(12, p_{2}, \ell\right)$;

(c) $\Gamma^{d}$ has a subgroup of type $\left(9, p_{1}, \ell\right)$;

(d) $\Gamma^{d}$ has subgroups of type $\left(18, p_{1}, \ell\right)$ and $\left(30, p_{2}, \ell\right)$; or

(e) $\Gamma^{d}$ has subgroups of type $\left(18, p_{1}, \ell\right),\left(20, p_{2}, \ell\right)$, and $\left(30, p_{3}, \ell\right)$,

then some finite quotient $\bar{\Gamma}$ of $\Gamma$ satisfies

$$
\left(H^{\text {ad }}\left(\overline{\mathbb{F}}_{\ell}\right)^{F}\right)^{\text {der }} \subset \bar{\Gamma} \subset H^{\text {ad }}\left(\overline{\mathbb{F}}_{\ell}\right)^{F},
$$

where $F$ is a Frobenius map and $H^{\text {ad }}$ is a simple adjoint algebraic group of type $\mathrm{G}_{2}, \mathrm{~F}_{4}, \mathrm{E}_{6}$, $\mathrm{E}_{7}$, or $\mathrm{E}_{8}$ respectively. In particular, in the first, second, and fifth case, $\bar{\Gamma}$ is simple.

Proof. Replacing $K$ by a finite extension, we may assume first that $G$ is split, and second that $\Gamma$ fixes a hyperspecial vertex of the building of $G$ over $K$ (see, e.g., [La], and the remark below). Thus, there exists a smooth group scheme $\mathcal{G}$ over the ring of integers $\mathcal{O}$ of $K$ whose generic fiber is $G$, whose special fiber is again reductive and connected, and such that $\Gamma \subset \mathcal{G}(\mathcal{O})$. The root datum of the special fiber of $\mathcal{G}$ is the same as that of $G$. Let $H:=\mathcal{G}_{\overline{\mathbb{F}}_{\ell}}$ denote the geometric special fiber. Thus, $\Gamma$ maps to $H\left(\overline{\mathbb{F}}_{\ell}\right)$ with finite image and pro- $\ell$ kernel. Replacing $\Gamma$ by its image in $H\left(\overline{\mathbb{F}}_{\ell}\right)$, by Lemma 10.1 and Lemma 10.3, we still have that $\Gamma^{d}$ has subgroups of the specified types. By Theorem 10.4, $H$ is almost simple of type $\mathrm{G}_{2}, \mathrm{~F}_{4}$, $\mathrm{E}_{6}, \mathrm{E}_{7}$, or $\mathrm{E}_{8}$ according as we are in case (a), (b), (c), (d), or (e). Assuming $p>3$, the image $\bar{\Gamma}$ of $\Gamma$ in $H^{\text {ad }}\left(\overline{\mathbb{F}}_{\ell}\right)$ again has the property that $\bar{\Gamma}^{d}$ has subgroups of the specified types. By [LP, Th. 0.5], it follows that either $\bar{\Gamma}$ satisfies the condition (10.6) for some Frobenius map or that $\bar{\Gamma}$ is contained in a proper algebraic subgroup of $I \subset H^{\text {ad }}$ with a component group $I / I^{\circ}$ whose order is bounded above by an absolute constant $A$. As $d>A$, it follows that $\bar{\Gamma}^{d} \subset I^{\circ}\left(\overline{\mathbb{F}}_{\ell}\right)$. If $N$ denotes the unipotent radical of $I^{\circ}$, the image of $\bar{\Gamma}^{d}$ in $(I / N)\left(\overline{\mathbb{F}}_{\ell}\right)$ contains a subgroup of type $(n, p, \ell)$. As $I / N$ is reductive of rank less than or equal to the rank $r$ of $H^{\text {ad }}$ (which equals the rank of $G$ ) and as $\operatorname{dim} I / N \leq \operatorname{dim} I<\operatorname{dim} H^{\text {ad }}=\operatorname{dim} G$, it follows that $I / N$ cannot be exceptional of rank $r$, which contradicts Theorem 10.4.

Remark: If $G$ is of type $\mathrm{G}_{2}, \mathrm{~F}_{4}$ or $\mathrm{E}_{8}$ then $G(K)$ is always split and simply connected. The profinite subgroup $\Gamma$ is contained in a maximal parahoric subgroup of $G(K)$. The quotient of this maximal parahoric subgroup by its pro- $\ell$ radical is a simply connected semisimple group $H$ of rank $r$ over the residual field of $K$. Let $\bar{\Gamma}$ be the projection of $\Gamma$ into $H$. If $\Gamma$ contains groups of type $(n, p, \ell)$ as specified in Theorem 10.5, then so does $\bar{\Gamma}$. By Theorem 10.4 $H$ must be of the same type as $G$. This shows that the maximal parahoric subgroup containing $\Gamma$ is hyperspecial.

\section{MAIN ThEOREM}

We are now ready to construct finite Galois groups over $\mathbb{Q}$. Let $r_{\Pi}: G_{\mathbb{Q}} \rightarrow G\left(\overline{\mathbb{Q}}_{\ell}\right)$ be the Galois representation attached to a self-dual cuspidal representation $\Pi$ constructed in Section 
6. Recall that $\Pi$ is constructed so that the image of $D_{q}$ in $\Gamma=r_{\Pi}\left(G_{\mathbb{Q}}\right)$ is a group of type $(2 n, p)$, denoted by $\Gamma_{2 n, p}$, and contained in $\Gamma^{d}$. By Theorem 9.2 and its corollaries, if $d>I(n)$ then $G=\mathrm{G}_{2}$ if $\Pi$ is a lift from $\mathrm{G}_{2}$ and $G=\mathrm{SO}_{2 n+1}$ if $\Pi$ is a lift from $\mathrm{Sp}_{2 n}$ and the local component $\Pi_{2}$ is the supercuspidal representation defined in Section 4, In particular, $\ell>2$ if $G=\mathrm{SO}_{2 n+1}$. If $G=\mathrm{G}_{2}$ then by Theorem $10.5 \Gamma$ has a quotient isomorphic to $\mathrm{G}_{2}\left(\mathbb{F}_{\ell^{k}}\right)$ (or a Ree group if $\ell=3$ ) for some $k$ provided that $d>A, B$ where $A$ and $B$ are in the statement of Theorem 10.5 .

Assume now that $G=\mathrm{SO}_{2 n+1}$ and that $\Pi$ is a lift from $\mathrm{Sp}_{2 n}$ and the local component $\Pi_{2}$ is the supercuspidal representation defined in Section 4 .

Theorem 11.1. Assume that $\ell>2$. There exists a function $d: \mathbb{N} \rightarrow \mathbb{N}$ such that if $\Pi_{q}$ is picked with $d>d(n)$ then $\Gamma=r_{\Pi}\left(G_{\mathbb{Q}}\right)$ has a quotient $\bar{\Gamma}$ such that

$$
\left(\mathrm{SO}_{2 n+1}\left(\overline{\mathbb{F}}_{\ell}\right)^{F}\right)^{\text {der }} \subset \bar{\Gamma} \subset \mathrm{SO}_{2 n+1}\left(\overline{\mathbb{F}}_{\ell}\right)^{F},
$$

where $F$ is a Frobenius map.

Proof. By enlarging the field $K$ we can assume that $G$ is split and that $\Gamma$ is contained in a hyperspecial maximal parahoric subgroup. This means that $G$ can be written as $G=$ $\mathrm{SO}(V, Q)$ where $V$ is a linear space over $K$ and $Q$ a split quadratic form, and there exists a lattice $L$ stabilized by $\Gamma$ such that $Q$ takes integral values on $L$. Moreover, if $\bar{V}$ is the reduction modulo $\ell$ of $L$, then the quadratic form $Q$ reduces modulo $\ell$ to a non-degenerate quadratic form $\bar{Q}$ on $\bar{V}$. In other words, the pair $(L, Q)$ defines a smooth group scheme over the ring of integers of $K$ whose generic fiber is $G$ and such that $H:=\operatorname{SO}(\bar{V}, \bar{Q})$ is the special fiber. Let $\bar{\Gamma}$ be the image of $\Gamma$ in $H\left(\overline{\mathbb{F}}_{\ell}\right)$. By [LP, Th. 0.5], it follows that either $\bar{\Gamma}$ satisfies the condition (11.2) for some Frobenius map or that $\bar{\Gamma}$ is contained in a proper algebraic subgroup of $I \subset H$ with a component group $I / I^{\circ}$ whose order is bounded above by an absolute constant $A(n)$. If we pick $d>A(n)$ then $\bar{\Gamma}^{d} \subset I^{\circ}\left(\overline{\mathbb{F}}_{\ell}\right)$. It follows that $\Gamma_{2 n, p}$ is contained in $I^{\circ}$. Under the action of $\Gamma_{2 n, p}$, the orthogonal space $\bar{V}$ decomposes as a sum of two irreducible mutually orthogonal representations of dimensions $2 n$ and 1 . This implies that the nilpotent radical of $I^{\circ}$ is trivial. Indeed, if $N$ is a non-trivial unipotent radical of $I^{\circ}$,then there exists a non-trivial subspace $\bar{U}$ of $\bar{V}$ fixed by $N$. Since $\Gamma_{2 n, p}$ normailizes $N, \bar{U}$ must be one of the two mutually orthogonal $\Gamma_{2 n, p}$-summands. By orthogonality, $N$ must preserve the other summand. Since that summand is also $\Gamma_{2 n, p}$-irreducible, $N$ must be trivial, a contradiction. Thus, $I^{\circ}$ is reductive. We claim that $\bar{V}$ is an irreducible $I^{\circ}$-module. If not, then $I^{\circ}$ admits a $2 n$-dimensional orthogonal representation such that there exists a Weyl group element in $I^{\circ}$ permuting transitively all weights. We need the following:

Lemma 11.3. Let $G$ be a connected reductive group over an algebraically closed field of characteristic $\neq 2$. Then $G$ has no irreducible orthogonal representations of even dimension such that there exists a Weyl group element permuting all weights.

Proof. Of course, we can assume that $G$ is semi-simple and that $G=G_{1} \times \cdots \times G_{k}$, a product of almost simple groups. If $V$ is a miniscule, self-dual representation of $G$ then $V=V_{1} \otimes \cdots \otimes V_{k}$ where $V_{i}$ are miniscule and self-dual representations of $G_{i}$. Of course, if $G$ contains a Weyl group element permuting all weights in $V$, then the same holds for all pairs $\left(G_{i}, V_{i}\right)$. The converse is true only if the dimensions of $V_{i}$ are pairwise relatively prime. If $G$ is simple, an argument in [KLS] shows that the only minuscule, self-dual representations with such Weyl 
group element is the standard representation of $\mathrm{Sp}_{2 n}$ and its Frobenius twists. (The list there includes also the standard representation of $\mathrm{SO}_{2 n}$, but that one can be excluded by a direct inspection.) Since even numbers are never pairwise relatively prime, we can conlude that $G$ is simple and that the representation is the standard representation of $\mathrm{Sp}_{2 n}$. This one is not orthogonal, however, and the lemma follows.

The lemma implies that $\bar{V}$ is an irreducible representation of $I^{\circ}$. Therefore, the pair $\left(I^{\circ}, \bar{V}\right)$ satisfies satisfies conditions of Lemma 7.2. It follows that $I^{\circ}=H$ or, if $n=3, I^{\circ}=\mathrm{G}_{2}\left(\overline{\mathbb{F}}_{\ell}\right)$. Since $\mathrm{G}_{2}$ has the trivial center, no outer automorphisms and it acts irreducibly on $\bar{V}$, any element of $I / I^{\circ}$ is represented by a scalar matrix. However, by Čebotarev's density theorem, any element in $\bar{\Gamma}$ has 1 as an eigenvalue. It follows that $I=I^{\circ}$. Since the image of the local decomposition group $D_{2}$ contains elements of order 2 which are not contained in $\mathrm{G}_{2}$ we see that $I$ cannot be $\mathrm{G}_{2}$. Thus we have $I^{\circ}=H$ in all cases. This is a contradiction. The theorem is proved with $d(n)=\max (A(n), I(n))$.

Summarizing, we have shown that $\bmod \ell$ reduction of the representations $r_{\Pi}$ give rise to $\mathrm{G}_{2}\left(\mathbb{F}_{\ell^{k}}\right)$ (or a Ree group if $\ell=3$ ), $\mathrm{SO}_{2 n+1}\left(\mathbb{F}_{\ell^{k}}\right)^{\text {der }}$ or $\mathrm{SO}_{2 n+1}\left(\mathbb{F}_{\ell^{k}}\right)$ as Galois group. In other words we have essentially proved the following theorem that we stated in the introduction:

Theorem 11.4. Let $t$ be a positive integer. We take $t$ to be even if $\ell=3$ in the first case below.

(1) Let $\ell$ be a prime. Then there exists an integer $k$ divisible by $t$ such that the simple group $\mathrm{G}_{2}\left(\mathbb{F}_{\ell^{k}}\right)$ appears as a Galois group over $\mathbb{Q}$.

(2) Let $\ell$ be an odd prime. Then there exists an integer $k$ divisible by $t$ such that the finite simple group $\mathrm{SO}_{2 n+1}\left(\mathbb{F}_{\ell^{k}}\right)^{\text {der }}$ or the finite classical group $\mathrm{SO}_{2 n+1}\left(\mathbb{F}_{\ell^{k}}\right)$ appears as a Galois group over $\mathbb{Q}$.

(3) If $\ell \equiv 3,5(\bmod 8)$, then there exists an integer $k$ divisible by $t$ such that the finite simple group $\mathrm{SO}_{2 n+1}\left(\mathbb{F}_{\ell^{k}}\right)$ der appears as a Galois group over $\mathbb{Q}$.

Proof. The divisibility of $k$ by $t$ follows from part (3) of Lemma 3.3. The remark following Lemma 3.3 shows that by taking $t$ even if $\ell=3$ in the first case eliminates Ree groups. It remains to deal with the third statement. Assume now that the Galois group given by part (2) is $\mathrm{SO}_{2 n+1}\left(\mathbb{F}_{\ell^{k}}\right)$. Then the subgroup $\mathrm{SO}_{2 n+1}\left(\mathbb{F}_{\ell^{k}}\right)^{\text {der }}$ of index 2 defines a quadratic field $K$. This field is unramified for all primes different from $2, \ell$ and $q$, since the same holds for the representation $r_{\Pi}$. Since the image of the inertia $I_{q}$ is of order $p$, it lands in the subgroup $\mathrm{SO}_{2 n+1}\left(\mathbb{F}_{\ell^{k}}\right)^{\text {der }}$. Thus, $K$ is unramified at $q$ also. Moreover, by Proposition 4.1, the image of the decomposition group $D_{2}$ is a group such that the quotient by its commutator is odd. Such group must be contained in the subgroup $\mathrm{SO}_{2 n+1}\left(\mathbb{F}_{\ell^{k}}\right)^{\text {der }}$. This shows not only that that $K$ is unramified at 2 but 2 splits in $K$. We remind the reader that the unique quadratic field ramified at $\ell$ and no other primes is $\mathbb{Q}(\sqrt{\ell})$ if $\ell \equiv 1(\bmod 4)$ and $\mathbb{Q}(\sqrt{-\ell})$ if $\ell \equiv 3(\bmod 4)$. However, since 2 splits in this field if and only if $\ell \equiv 1,7(\bmod 8)$ we see that if $\ell \equiv 3,5$ $(\bmod 8)$ the Galois group constructed in part $(2)$ is in fact $\mathrm{SO}_{2 n+1}\left(\mathbb{F}_{\ell}^{k}\right)^{\text {der }}$.

\section{On FUTURE DIRECTIONS}

One difficulty that we needed to address in this paper came from the fact that the group $\mathrm{GL}_{2 n+1}\left(\mathbb{Q}_{p}\right)$ has no self-dual supercuspidal representation unless $p=2$. In order to construct 
Galois groups of type $\mathrm{B}_{n}$ this problem was resolved by introducing the self-dual supercuspidal representation $\Pi_{2}$ of $\mathrm{GL}_{2 n+1}\left(\mathbb{Q}_{2}\right)$ whose parameter contains a Jordan subgroup of $\mathrm{SO}_{2 n+1}(\mathbb{C})$. For Galois groups of type $\mathrm{G}_{2}$ the construction is based on a technical improvement of Theorem 1.1 due to Shin, which is based on the fundamental lemma for unitary group. Another way, which avoids the use of the fundamental lemma, would be to pick $\Pi_{2}$ so that its parameter comes from the Jordan subgroup in $G_{2}$. More precisely the parameter of $\Pi_{2}$ should be the homomorphism $\phi_{2}: W_{\mathbb{Q}_{2}} \rightarrow \mathrm{G}_{2}$ described in Proposition 4.2. In order to obtain a global lift from $\mathrm{G}_{2}$ to $\mathrm{GL}_{7}$ with this $\Pi_{2}$ as a local component one would need to complete the following (doable) program:

- Define, via induction from open compact subgroup, a generic supercuspidal $\sigma_{2}$ representation of $\mathrm{G}_{2}\left(\mathbb{Q}_{2}\right)$ corresponding to the parameter $\phi_{2}$.

- Compute the theta lift of $\sigma_{2}$ to $\operatorname{PGSp}_{6}\left(\mathbb{Q}_{2}\right)$.

- Show, using the method of $\left[\mathrm{Sa2}\right.$, that the further lift to $\mathrm{GL}_{7}\left(\mathbb{Q}_{2}\right)$ is $\Pi_{2}$.

A construction of supercuspidal representations attached to parameters arising from Jordan subgroups is a subject of the forthcoming paper by Gross and Reeder [GR]. The completion of the three step program would give Galois groups of type $\mathrm{G}_{2}$ except in the residual characteristic 2 without using results of Shin [Sh]. There is yet another approach which removes the restriction $\ell \neq 2$, but introduces a different conjecture. Let $G$ denote $\operatorname{Sp}_{2 n}$ or $\mathrm{G}_{2}$. Then using the trace formula it is possible to show that there exist a cuspidal automorphic representation $\sigma$ of $G(\mathbb{A})$ unramified at all primes different from $\ell, q$ and such that

- $\sigma_{\infty}$ is a discrete series representation with a large (unspecified) parameter (weight).

- $\sigma_{q}$ is a specified supercuspidal representation.

- $\sigma_{\ell}$ is the Steinberg representation.

Assuming that $\sigma$ is globally generic then $\Pi$, the lift of $\sigma$ to $\mathrm{GL}_{2 n+1}(\mathbb{A})$, is automatically cuspidal and has a discrete series representation at one local place, since $\Pi_{\ell}$ is the Steinberg representation of $\mathrm{GL}_{2 n+1}\left(\mathbb{Q}_{\ell}\right)$. (We use here that the theta lift of the Steinberg representation of $\mathrm{G}_{2}\left(\mathbb{Q}_{\ell}\right)$ is the Steinberg representation of $\mathrm{PGSp}_{6}\left(\mathbb{Q}_{\ell}\right)$, see $[\mathrm{GrS}]$.) Since matrix coefficients of the Steinberg representation are in $L^{1+\epsilon}(G)$ and therefore not integrable, we note that the method of Poincaré series cannot be used to construct such $\sigma$.

In principle our method could be extended to other groups. The main limitation at the moment is the lack of $\ell$-adic representations attached to automorphic representations. If we assume, for example, that one can attach a 26 -dimensional $\ell$-adic representation to an algebraic automorphic form of the exceptional group $\mathrm{F}_{4}$ then we would be able to construct finite groups of type $\mathrm{F}_{4}$ as Galois groups over $\mathbb{Q}$. Indeed, to this end one would pick a cuspidal automorphic form $\sigma$ of $\mathrm{F}_{4}$ such that for two primes $p_{1}$ and $p_{2}$ the local components $\sigma_{p_{1}}$ and $\sigma_{p_{2}}$ are tame supercuspidal representations whose parameters have groups of type $\left(8, p_{1}\right)$ and $\left(12, p_{2}\right)$, respectively, as the image. Thus, if the two parameters at $p_{1}$ and $p_{2}$ are picked so that the images of the local decomposition groups are deeply embedded, then the results of Section 10 imply that the restriction modulo $\ell$ of the $\ell$-adic representation attached to $\sigma$ will give finite groups of type $\mathrm{F}_{4}\left(\ell^{k}\right)$ as Galois groups over $\mathbb{Q}$.

\section{REFERENCES}

[Ca] R. Carter: Finite Groups of Lie Type, Wiley Classics Library, New York, 1993. 
[Cl] L. Clozel, Représentations galoisiennes associées aux représentations automorphes autoduales de GL $(n)$. Inst. Hautes Études Sci. Publ. Math. No. 73 (1991), 97-145.

[CKPS] J. Cogdell, H. Kim, I. Piatetski-Shapiro and F. Shahidi, Functoriality for the classical groups. Publ. Math. Inst. Hautes Études Sci. No. 99 (2004), 163-233.

[DR] S. DeBacker and M. Reeder, Depth zero supercuspidal L-packets and their stability. To appear in Annals of Math.

[Ga] W. T. Gan, Exceptional Howe correspondences over finite fields. Compositio Math. 118 (1999), 323344 .

[GaS1] W. T. Gan and G. Savin Real and global lifts from PGL 3 to G. Inter. Math. Res. Not. 50 (2003), 2699-2724.

[GaS2] W. T. Gan and G. Savin Endoscopic lifts from $\mathrm{PGL}_{3}$ to $\mathrm{G}_{2}$. Compositio Math. 140 (2004), $793-808$.

[GRS] D. Ginzburg, S. Rallis and D. Soudry, A tower of theta correspondences for $G_{2}$. Duke Math. J. 88 (1997), 537-624.

[GR] B. H. Gross and M. Reeder, arithmetic invariants of discrete Langlands parameters. In preparation.

[GrS] B. H. Gross and G. Savin, Motives with Galois group of type $G_{2}$ : an exceptional theta correspondence. Compositio Math. 114 (1998), 153-217.

[HT] M. Harris, R. Taylor. The geometry and cohomology of some simple Shimura varieties. Annals of Mathematics Studies, 151. Princeton University Press, Princeton, NJ, 2001. viii+276 pp.

[Ha] M. Harris. Potential automorphy of odd-dimensional symmetric powers of elliptic curves, and applications. to appear in Algebra, Arithmetic, and Geometry: Manin Festschrift (Birkhuser, in press).

[HPS] J. S. Huang, P. Pandžić and G. Savin, New dual pair correspondences. Duke Math. J. 82 (1996), 447-471.

[Hu1] James E. Humphreys: Linear Algebraic Groups. Graduate Texts in Mathematics, 21. Springer-Verlag, New York, 1975.

[Hu2] James E. Humphreys: Conjugacy classes in semisimple algebraic groups. Mathematical Surveys and Monographs, 43. American Mathematical Society, Providence, RI, 1995.

[JS1] D. Jiang, D. Soudry. The local converse theorem for $\mathrm{SO}(2 n+1)$ and applications. Ann. of Math. (2) 157 (2003), no. 3, 743-806.

[JS2] D. Jiang, D. Soudry. Lecture at the workshop on Automorphic Forms, Geometry and Arithmetic. Oberwolfach, February 2008. Announcement available at http://www.mfo.de/

[KLS] C. Khare, M. Larsen and G. Savin, Functoriality and the inverse Galois problem. Compositio Math. 144 (2008), 541-564.

[KT] A. I. Kostrikin and P. H. Tiep: Orthogonal Decompositions and Integral Lattices, De Gruyter Expositions in Mathematics 15, Walter de Gruyter, Berlin - New York, 1994.

[KW] Chandrashekhar Khare and Jean-Pierre Wintenberger, Serre's modularity conjecture (I), preprint available at http://www.math.utah.edu/ shekhar/papers.html

[La] Michael Larsen, Maximality of Galois actions for compatible systems, Duke Math. J. 80 (1995), no. $3,601-630$.

[LP] Michael Larsen and Richard Pink, Finite subgroups of algebraic groups, preprint available at http://www.math.ethz.ch/ ${ }^{\sim}$ pink/publications.html

[MaS] K. Magaard and G. Savin, Exceptional theta correspondences. Compositio Math. 107 (1997), 89-123.

[Moy] A. Moy, The irreducible orthogonal and symplectic Galois representations of a p-adic field (the tame case). Journal of Number Theory 10 (1984), 341-344.

$[\mathrm{Mu}] \quad$ G. Muić, The unitary dual of p-adic $G_{2}$. Duke Math. J. 90 (1997), 465-493.

[Sa1] G. Savin, K-types of minimal representations (p-adic case). Glasnik Mat. Vol. 31(51) (1996), 93-99.

[Sa2] G. Savin, Lifting of generic depth zero representations of classical groups. J. of Algebra 319 (2008), 3244-3258.

[Sh] Sug Woo Shin, Odd dimensional Galois representations arising from some compact Shimura varieties. Preprint, IAS, 2008.

[Ta] M. Tadić. Representations of p-adic symplectic groups. Compositio Math. 90 (1994), $123-181$.

[Ty] R. Taylor, Galois representations. Ann. Fac. Sci. Toulouse Math. 13 (2004), 73-119.

[W] Gabor Wiese. On projective linear groups over finite fields as Galois groups over the rational numbers. preprint available at http://xxx.lanl.gov/abs/math.NT/0606732 
E-mail address: shekhar@math.utah.edu

Department of Mathematics, UClA, Los Angeles CA 90095-1555, U.S.A.

E-mail address: larsen@math.indiana.edu

Department of Mathematics, Indiana University, Bloomington, IN 47405, U.S.A.

E-mail address: savin@math.utah.edu

Department of Mathematics, University of Utah, 155 South 1400 East, Room 233, Salt Lake City, UT 84112-0090, U.S.A. 